\title{
GRACE Accelerometer Data Transplant
}

Tamara Bandikova $^{\mathrm{a}, *}$, Christopher McCullough $^{\mathrm{a}}$, Gerhard L. Kruizinga $^{\mathrm{a}}$, Himanshu Save $^{\mathrm{b}}$, Bruno Christophe $^{\mathrm{c}}$

${ }^{a}$ Jet Propulsion Laboratory, California Institute of Technology, 4800 Oak Grove Dr, 91109 Pasadena, CA, USA

${ }^{b}$ University of Texas at Austin, Center for Space Research, 3925 West Braker Lane, 78759 Austin, Texas ${ }^{c}$ DPHY, ONERA, Université Paris Saclay - F-92322 Chatillon, France

\begin{abstract}
In the last few months of GRACE (Gravity Recovery and Climate Experiment) mission operation, the accelerometer (ACC) aboard GRACE-B was turned off due to battery problems. In order to compute a gravity field model for these months, a data processing strategy was developed to retrieve the missing information, the so called Accelerometer data transplant. The ACC data transplant uses linear accelerations as measured by the GRACE-A accelerometer to generate the missing GRACE-B ACC data. A simple method of ACC data transplant was presented by Save et al. (2006), which only applies attitude and time correction due to orbit separation. In this paper we present an improved method of the ACC data transplant, which also includes thruster spike correction. The thruster spikes are residual linear accelerations occurring at each thruster firing caused by thruster imperfections and misbalance. We provide detailed description of the thruster spike model, which was derived analytically based on the ACC transfer function and $10 \mathrm{~Hz}$ ACC data. Using the transplanted ACC data we computed the gravity field models for the months with missing GRACE-B ACC data (November 2016 - June 2017). We show that the gravity field model based on in the improved ACC data transplant has significantly reduced noise, especially for degrees beyond the first orbital resonance, when compared to the simple transplant method. The improved ACC transplant became therefore a part of the processing standards for the upcoming JPL RL06 gravity solution.
\end{abstract}

Keywords:

Gravity Recovery and Climate Experiment; GRACE; Accelerometer; Gravity field, Thruster spikes; Data transplant

\footnotetext{
${ }^{*}$ Corresponding author

Email addresses: tamara.bandikova@jpl.nasa.gov (Tamara Bandikova), Christopher.Mccullough@jpl.nasa.gov (Christopher McCullough), Gerhard.L.Kruizinga@jpl.nasa.gov (Gerhard L. Kruizinga), save@csr.utexas.edu (Himanshu Save), bruno.christophe@onera.fr (Bruno Christophe)
} 


\section{Introduction}

In November 2017, the Gravity Recovery and Climate Experiment (GRACE) (Tapley et al., 2004) ended its mission. The final year was especially challenging for both mission operation and science data processing. The aging of the spacecraft affected battery health and fuel reserve the most. After losing several battery cells on GRACE-B, there was not enough voltage to nominally operate the spacecraft. Several measures were taken to reduce battery load (Herman et al., 2012, Witkowski and Massmann, 2016, 2017). One of the measures was turning off the accelerometer (ACC) on GRACE-B since September 2016.

The ACC measurement from both satellites, however, is necessary for the gravity field recovery. The accelerometer provides information about the non-gravitational forces acting on the spacecraft, such as atmospheric drag, solar radiation pressure and Earth albedo, at the level of precision of $10^{-10} \mathrm{~ms}^{-2} / \sqrt{H z}($ Touboul et al. 2004). In order to obtain orbit perturbations caused solely by the gravitational attraction of the Earth mass, the effect of non-gravitational forces have to be reduced from the original measurement, i.e. microwave inter-satellite ranging and GPS observations (Kang et al., 2006). Estimating the non-gravitational accelerations based on atmospheric and other models, however, does not provide sufficient accuracy. Klinger and Mayer-Gürr (2016) clearly demonstrated that for lower satellites' altitudes $(<410 \mathrm{~km})$ large discrepancies between the modeled and measured accelerations exist, which are mainly caused by imperfections in the atmospheric density models. Hence in order to obtain the gravity field solutions for the months with single ACC operation, a new data processing strategy had to be developed to retrieve the missing information, the so called ACC data transplant.

The ACC data transplant uses GRACE-A ACC measurement to generate the missing GRACE-B ACC data. The ACC data transplant was already studied by Save et al. $(2006)$ as the ACC data was missing for several weeks in 2002 and 2003. After the GRACE-B accelerometer was turned off in 2016, the ACC data transplant became part of the official solution for the gravity field models starting from November 2016 (Save, 2016). In the solution presented by Save, only attitude and time correction is applied to the GRACE-A ACC data to generate the missing GRACE-B data.

However, the accelerometer senses not only the accelerations caused by non-gravitational forces, but also linear accelerations caused by spacecraft operation and environment, such as residual linear accelerations due to thruster firing, twangs or heater switching spikes (Flury et al., 2008, Peterseim, 2014). These disturbing accelerations are unique for each spacecraft. While twangs and heater switching spikes are filtered out during ACC Level-1A to Level-1B processing ( $\mathrm{Wu}$ et al., 2006), the residual linear acceleration due to thruster firings ("thruster spikes") remain in the ACC data even after the $35 \mathrm{mHz}$ low-pass filtering. Meyer et al. (2012) proved that these thruster spikes (mostly reaching up to a few hundreds $n m \cdot s^{-2}$, but is some cases exceeding $1000 \mathrm{~nm} \cdot \mathrm{s}^{-2}$ ) are real linear accelerations which have to be taken into account for orbit determination. Therefore, if not corrected, the thruster spikes are the largest high-frequency error source in the transplanted ACC data.

We present a new method for the modeling of the residual linear accelerations due to thruster firings. Our thruster spike model is based on an analytic function obtained by inverse Laplace transform of the ACC transfer function. The model is determined for each thruster pair and for the whole scale of possible thruster firing duration times (30-1000 ms) based on the fit to the raw unfiltered ACC measurement data, the $10 \mathrm{~Hz}$ ACC Level-1A data. This is a new approach as most of the previously developed thruster spikes models were based on ACC Level-1B data, i.e. $35 \mathrm{mHz}$ low-pass filtered data with 1s sampling (Meyer et al., 2012; Tregoning et al. 2013) and thus do not reflect the true nature of the thruster spikes. The thruster spike model developed by McCullough et al. (2015) was already based on the Level-1A ACC data and the knowledge of accelerometer transfer function, however, the model was created for averaged acceleration profiles and for yaw and roll thruster pairs only.

The goal of this paper is to present an improved ACC data transplant method which includes the thruster spike correction in addition to the attitude and time correction. The newly generated ACC data are validated for selected months, in which accelerometers on both satellites were turned on. We provide numerical comparison of our new method to the original method presented by Save et al. (2006). Finally, we present the gravity field solutions for all months with single ACC operation, i.e. November 2016 - June 2017.

In Section 2 we provide a brief overview about the accelerometer data and we focus on characterization of the thruster spikes. In Section 3 we describe in detail the accelerometer data transplant and all the necessary 
corrections. In Section 4 we present validation of the ACC data transplant based on comparison with original GRACE-B data. And finally in Section 5 we show the gravity field solutions for the months with missing GRACE-B ACC data.

\section{Accelerometer data}

The SuperSTAR three-axis capacitive accelerometer (ACC) manufactured by the Office National d'Etudes et de Recherches Aerospatiales (ONERA) (Touboul et al., 1999b) is mounted at the center of mass (CoM) of each satellite. The sensor unit consists of a titanium proof-mass with dimensions of $40 \mathrm{x} 40 \mathrm{x} 10 \mathrm{~mm}^{3}$ surrounded by an electrode cage. The acceleration measurement is performed by measuring electrostatic forces applied by the cage electrodes to keep the proof-mass motionless with respect to a cage. These forces are proportional to the difference of acceleration acting on the cage and on the proof-mass. Since the accelerometer is located in satellite's CoM, the measured acceleration is proportional to non-gravitational forces acting on the satellite, such as air drag, solar radiation pressure and Earth albedo (Touboul et al., 2004, Frommknecht, 2008, Peterseim, 2014).

The air drag dominates the along-track component of the linear acceleration. The solar radiation pressure is strongest in the radial component. The Earth albedo represents the smallest non-gravitational force acting on the GRACE spacecraft (Klinger and Mayer-Gürr, 2016). The magnitude of these forces depends especially on the atmospheric density, solar flux, satellite's attitude, satellite's surface characteristics and Earth's surface properties. The linear acceleration can be determined with a precision of $10^{-10} \mathrm{~ms}^{-2} / \sqrt{\mathrm{Hz}}$ for the high sensitive axes (radial and along-track axes) and $10^{-9} \mathrm{~ms}^{-2} / \sqrt{\mathrm{Hz}}$ for the less sensitive axis (cross-track axis) (Flury et al. 2008).

The measured linear acceleration is disturbed by high-frequency signals caused by satellite's operation or environment, such as thruster spikes (see Section 2.1), twangs (see Section 2.2), heater switching spikes or magnetic torquer spikes (see Section 2.3). These high-frequency signals are unique for each satellite. The main focus in this paper is on the thruster spikes as they are the most critical for the ACC data transplant.

The accelerometer also provides information about the angular acceleration of the spacecraft, which can be used for attitude determination (Klinger and Mayer-Gürr, 2014, Sakumura et al., 2017). The angular acceleration, however, cannot be transplanted from the measurement of the other satellite, because it purely reflects the attitude variations of the individual satellite. For this reason, in the following, we only focus on the linear acceleration.

The ACC data are stored in ACC1A and ACC1B data products, which refer to the respective GRACE Level-1A and Level-1B data (Bettadpur, 2012). Briefly summarized, the ACC1A data are the raw, $10 \mathrm{~Hz}$ data, given in Accelerometer reference frame (AF) and in receiver clock time. The ACC1B are $35 \mathrm{mHz}$ low-pass filtered, $1 \mathrm{~Hz}$ data, given in the Science reference frame (SRF) and in GPS time. The ACC1A to ACC1B processing is described in ( $\mathrm{Wu}$ et al., 2006). The reference frames are sketched in Figure 1, for more details check (Bettadpur. 2012). For better understanding, when possible, we refer to the accelerometer axes as along-track, cross-track and radial. 


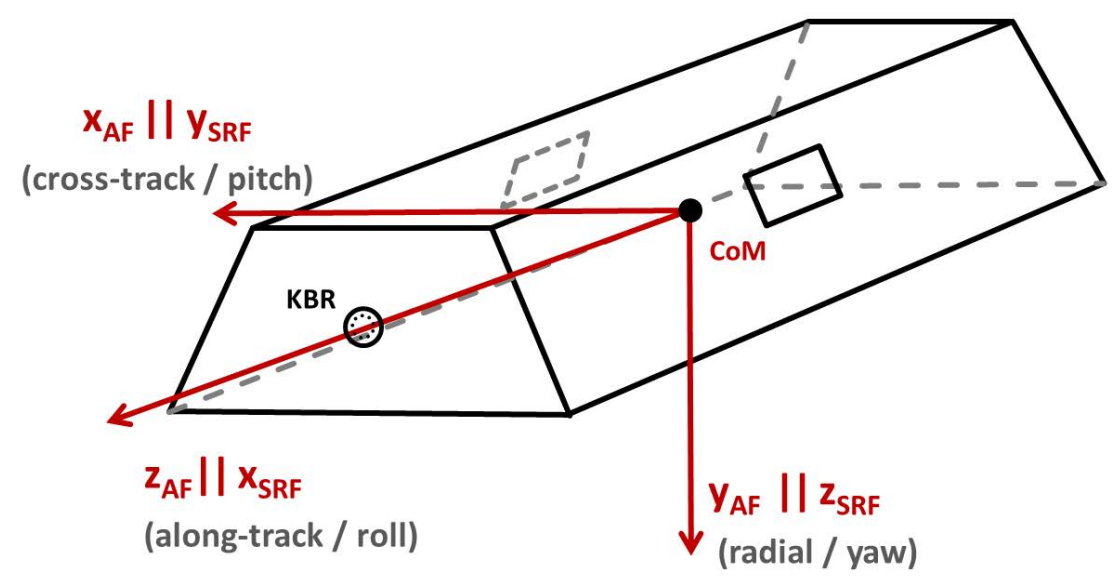

Figure 1: Accommodation of the Accelerometer reference frame (AF) and the Science reference frame (SRF) in the GRACE satellite body

\subsection{Thruster spikes}

The thruster spikes are residual linear accelerations caused by imperfections of attitude control thrusters. There are twelve $10 \mathrm{mN}$ cold gas attitude control thrusters onboard each satellite (Schelkle, 2000; Bettadpur, 2012). The thrusters are designed to operate in pairs in order to control only the angular acceleration about the \pm roll, \pm pitch and \pm yaw axis of the spacecraft, cf. Figure 2, If the thrusters were perfect, there would be no linear acceleration sensed by the accelerometer. Unfortunately, this is not the case. Due to possible misalignment of the thruster pair, applied force imbalance, differences in thruster reaction time, differences in feed pressure for a thruster pair, and/or misalignment of the ACC proof-mass from the center of mass of the spacecraft, the thruster firings cause linear acceleration as well.

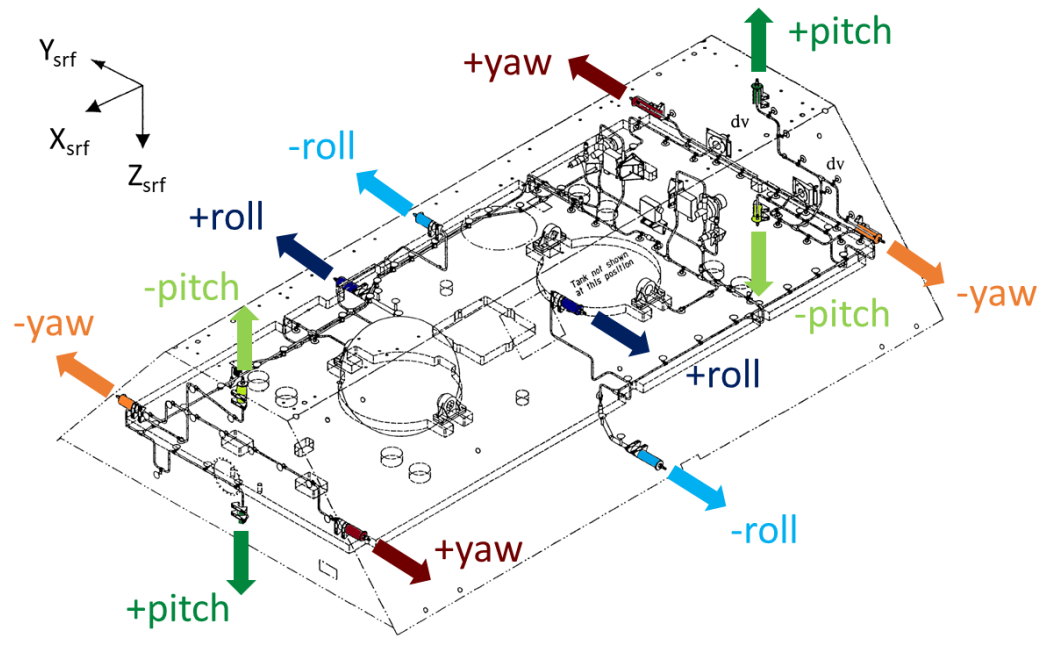

Figure 2: Attitude control thrusters onboard GRACE satellite, source: Schelkle (2000)

Figure 3 shows a sample of raw $10 \mathrm{~Hz}$ linear acceleration superimposed by the thruster spikes. They occur immediately after the thruster firing and last for no more than one or two seconds. Figure 4 shows one particular type of thruster spike caused by a $100 \mathrm{~ms}$ positive roll thruster firing. The magnitude of the thruster spikes can exceed $1000 \mathrm{~nm} \cdot \mathrm{s}^{-2}$. There is a slight delay between the actual thruster activation and the reaction of the accelerometer of approx. 0.14 s due to the applied Butterworth filter in the onboard data processing chain. Figure 4 shows approx. 180 thruster spikes of the same type plotted on top of each other. Obviously, thruster spikes of one particular type are very consistent, which allows for the thruster spike modeling. 


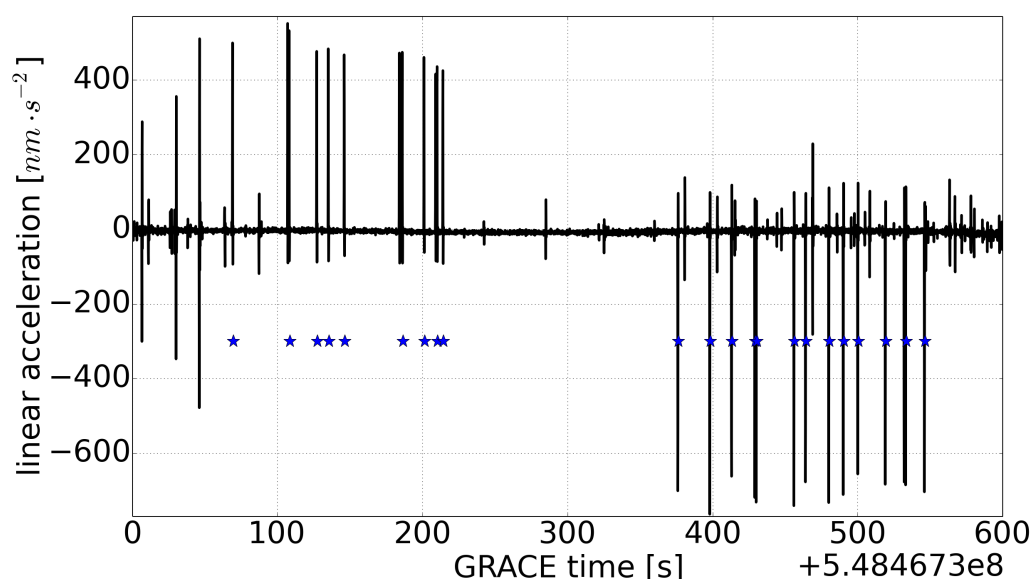

Figure 3: A sample of the raw $10 \mathrm{~Hz}$ linear acceleration (cross-track component) with clearly visible thruster spikes, one of the most dominant high-frequency signals. The blue stars indicate the time when the attitude control thrusters were activated

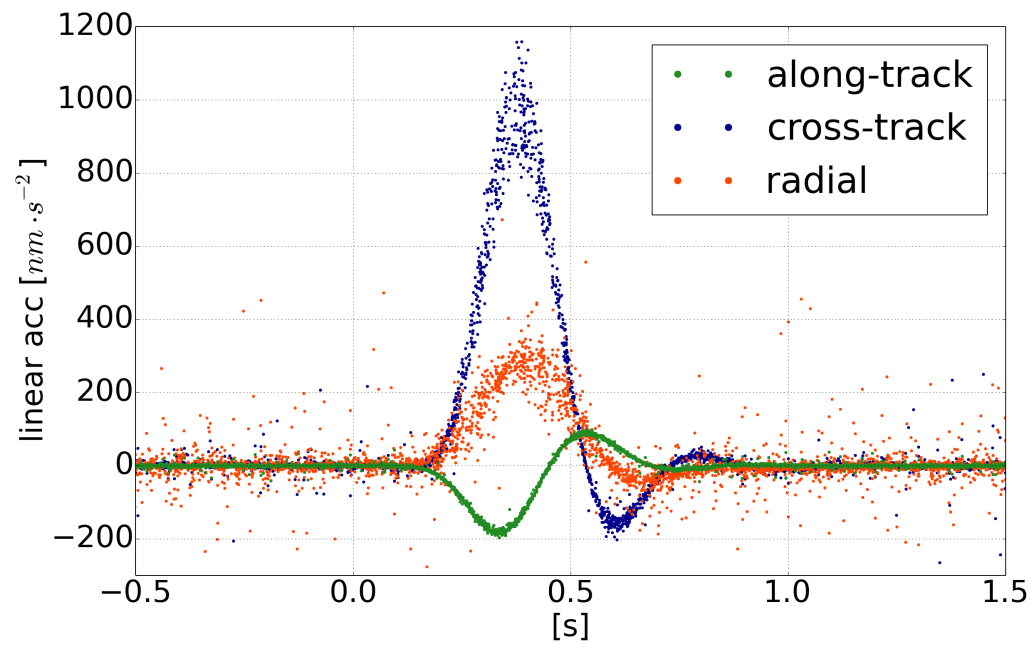

Figure 4: Thruster spikes caused by $100 \mathrm{~ms}$ + roll firing as seen in all three components of the raw $10 \mathrm{~Hz}$ linear acceleration on GRACE-A on 2017-05-19. There are approx. 180 spikes plotted on top of each other.

As the thruster imperfections are different for each thruster pair, the shape and amplitude of the thruster spike varies for each thruster pair $( \pm$ roll, \pm pitch, \pm yaw $)$, for each xyz component, for each firing duration (from $30 \mathrm{~ms}$ to $1000 \mathrm{~ms}$ ) and also are different for each spacecraft, cf. Figure 5.

The thrusters are activated on average about 1000 times a day. The most frequent are \pm yaw thruster firings, least frequent pitch thruster firings. The firing duration varies from $30 \mathrm{~ms}$ to $1000 \mathrm{~ms}$. The thrusters are the secondary attitude actuators for GRACE and they are activated only when the control torques generated by the magnetic torquers do not suffice to maintain the desired attitude (Herman et al., 2004). For this reason, the geographical location of the thruster activations is strongly correlated with the direction of Earth's magnetic field lines, see Figure 6.

The thrusters operate based on an adaptive strategy using a pulse width modulator (PWM). This means the length of the pulse depends upon the strength and the results of the preceding thrusts (Herman et al., 2004). The PWM, however, had been turned off since December 2014 on both satellites to reduce the fuel consumption. As a result, the thrusters' firing duration is shorter, but the thrusters are activated more often. Except for large attitude maneuvers, the firing duration is constant. Table 1 shows the frequency of the thruster activations for the preferred firing duration. In case of roll and yaw thrusters, the firing duration is the same for more than $93 \%$ of all thruster activations. 
More importantly, after the PWM was turned off, the thruster spikes became very consistent in shape and amplitude. Figure 7 shows the thruster spikes for one day in 2008 and 2017. In the early years, when the PWM was turned on, the thruster spikes had different directions and amplitude even for the same type of thrust. Hence it would be practically impossible to model these thruster spikes precisely. But after the PWN was turned off, the spikes are now very consistent in shape, amplitude and direction, which allows us to model and to reconstruct them well. This is the reason, why we use only data from 2015-2017 as basis to find the thruster spike model. We don't use any data prior that period, because of the inconsistency of the thruster spikes.

Figure 8 shows an example of one thruster spike as seen in both ACC1A and ACC1B data. Due to its asymmetric shape, the thruster spike is not filtered out by the $35 \mathrm{mHz}$ low-pass filter, but it is smeared over approx. $70 \mathrm{~s}$. The energy of the pulse is preserved. 


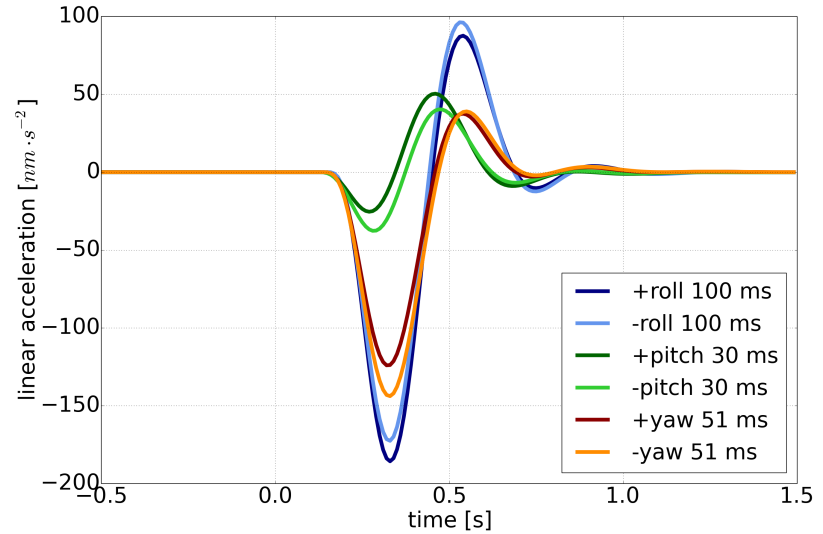

(a) along-track

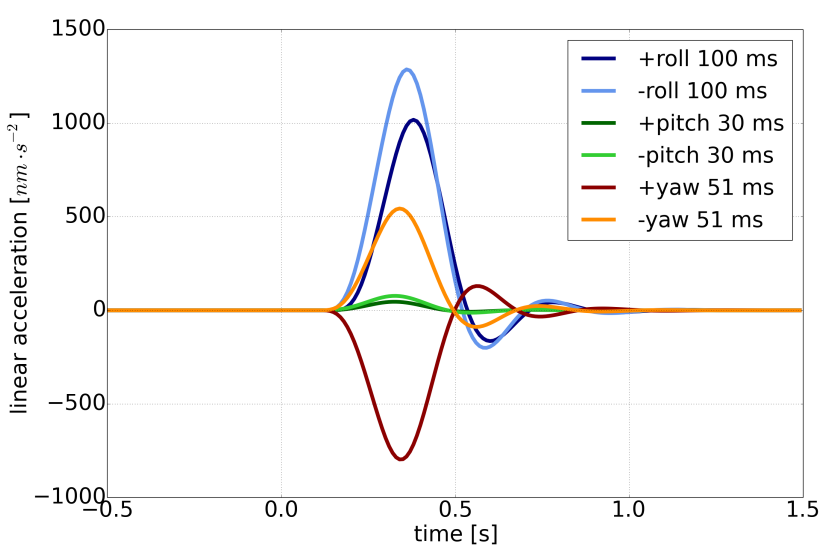

(c) cross-track

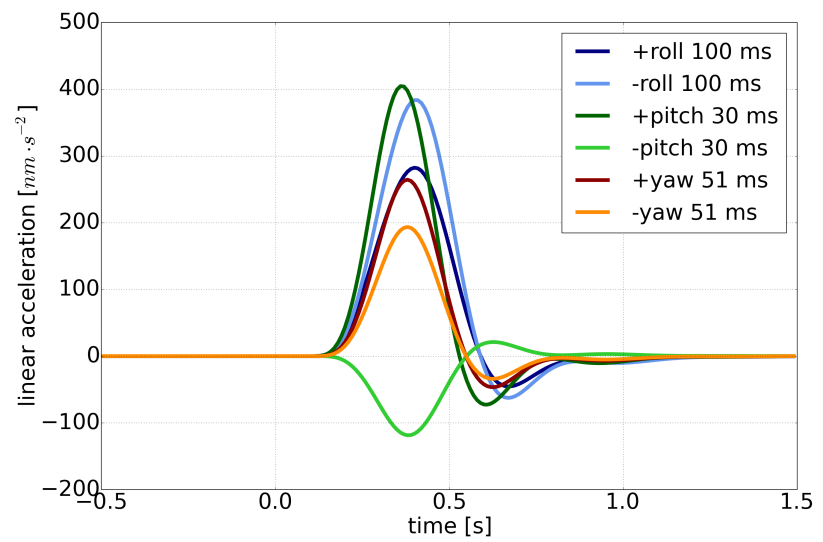

(e) radial

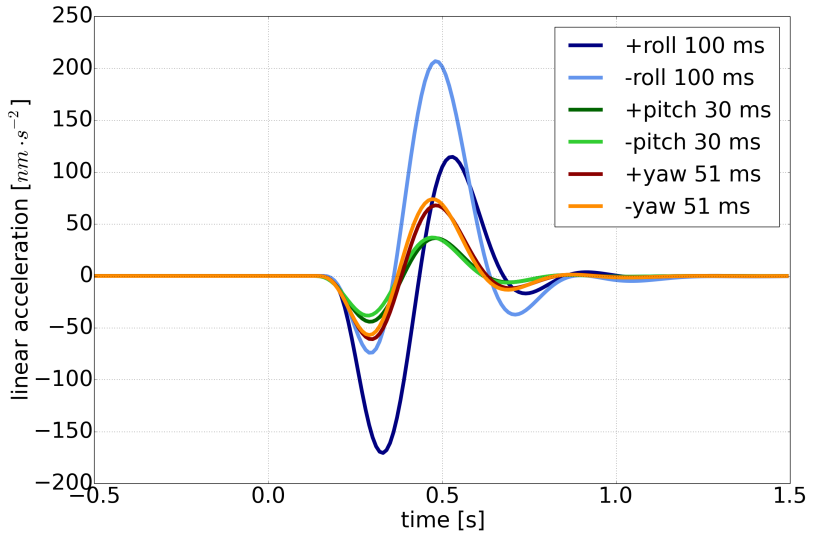

(b) along-track

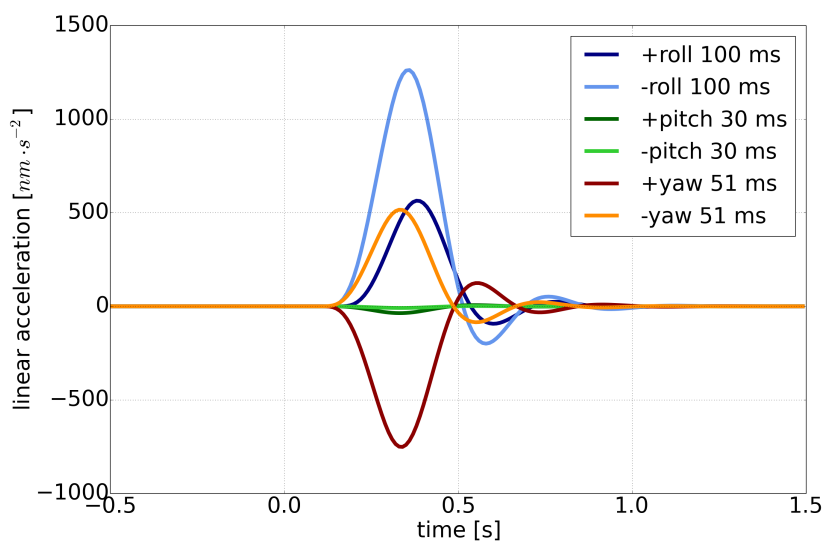

(d) cross-track

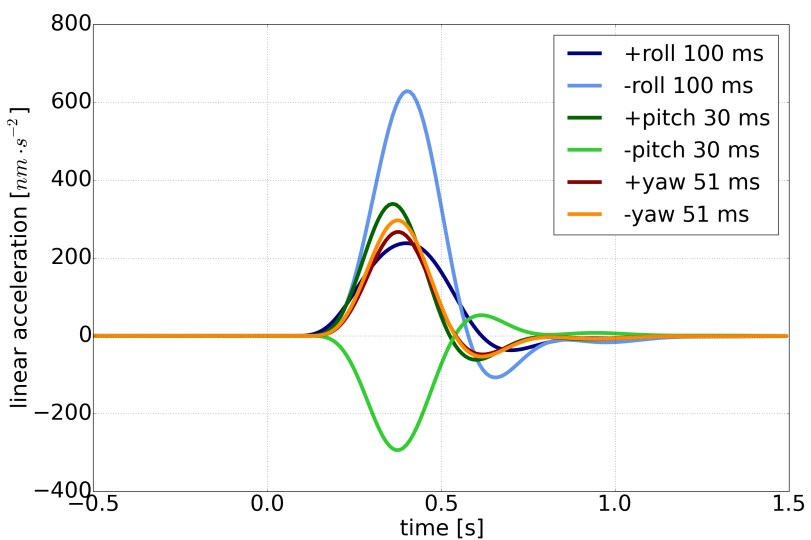

(f) radial

Figure 5: Thruster spikes for the most frequent firing durations for each thruster pair on GRACE-A (left column) and GRACE-B (right column) 


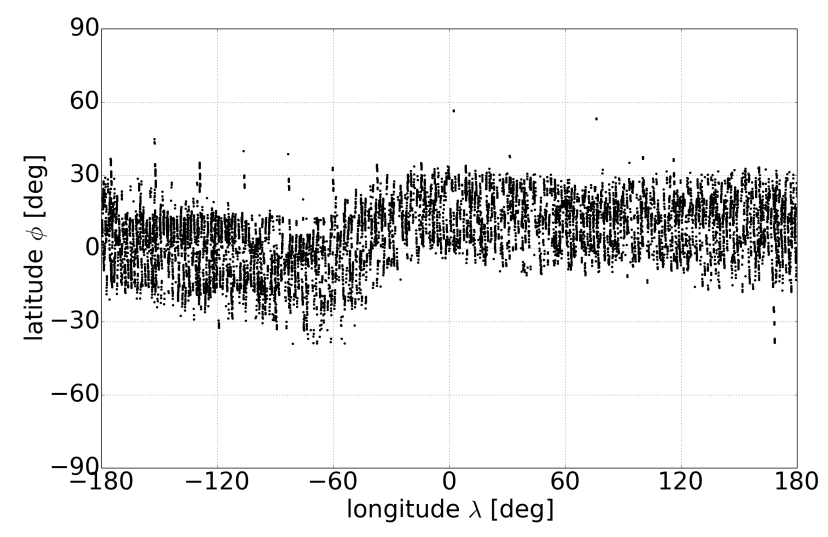

(a) roll

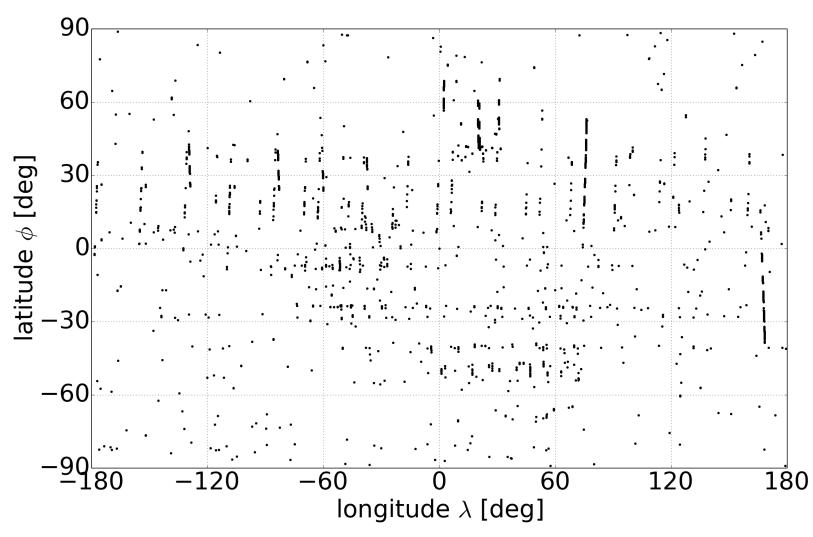

(b) pitch

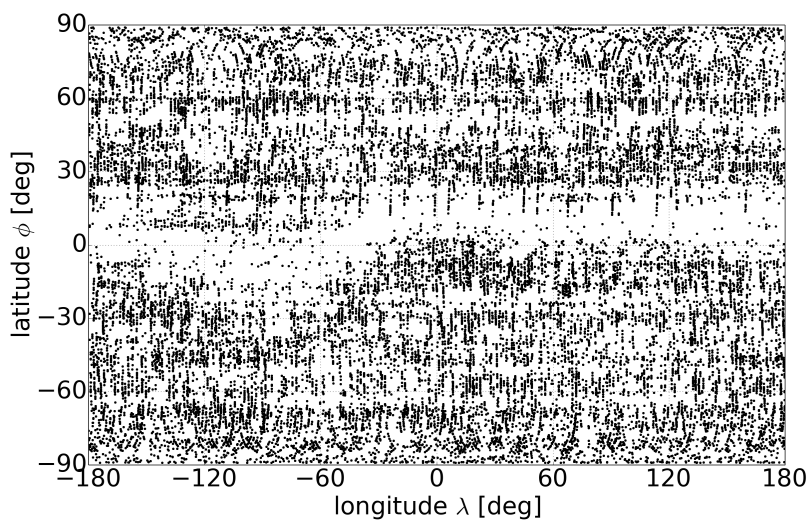

(c) yaw

Figure 6: Geographical location the GRACE-A roll, pitch and yaw thruster activations in January 2017. The roll thrusters are activated mainly along the geomagnetic equator and the yaw thrusters in high latitude, because in those regions one of the magnetic torquer rods is parallel to the Earth's magnetic field lines and hence the generated control torque is insufficient

Table 1: Frequency of thruster activations with the specific firing duration. The values are based on data from Nov 2016 to Jun 2017 during science mode operation. In other words, e.g. the GRACE-A + roll thruster pair is activated for $100 \mathrm{~ms}$ in $98 \%$ of all cases

\begin{tabular}{lll|ll} 
& GRACE-A & & GRACE-B & \\
+roll & $100 \mathrm{~ms}$ & $98 \%$ & $100 \mathrm{~ms}$ & $80 \%$ \\
-roll & $100 \mathrm{~ms}$ & $97 \%$ & $100 \mathrm{~ms}$ & $90 \%$ \\
+pitch & $30,31 \mathrm{~ms}$ & $76 \%$ & $30,31,32 \mathrm{~ms}$ & $61 \%$ \\
-pitch & $30,31 \mathrm{~ms}$ & $79 \%$ & $30,31,32 \mathrm{~ms}$ & $60 \%$ \\
+yaw & $51,52 \mathrm{~ms}$ & $94 \%$ & $51,52 \mathrm{~ms}$ & $93 \%$ \\
-yaw & $51,52 \mathrm{~ms}$ & $94 \%$ & $51,52 \mathrm{~ms}$ & $93 \%$
\end{tabular}




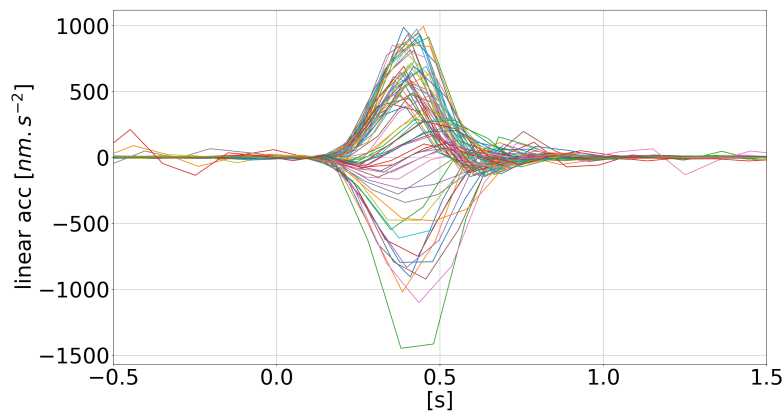

(a)

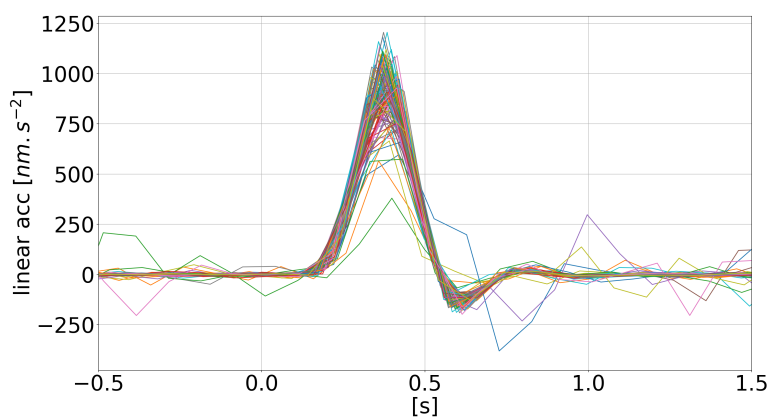

(c)

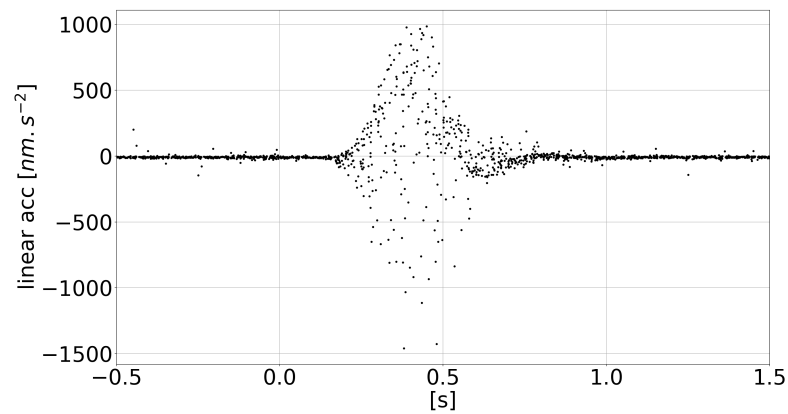

(b)

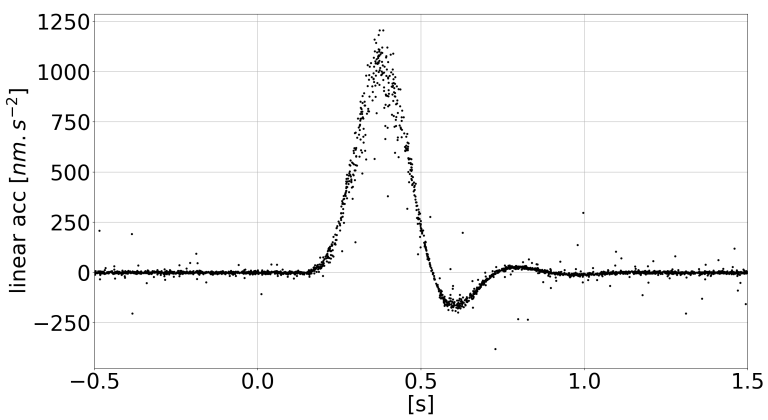

(d)

Figure 7: Demonstration of the effect of Pulse Width Modulator (PWM) being turned on (a,b) and turned off (c,d). The figures show thruster spikes (plotted on top of each other) in ACC1A data induced by positive roll firings for GRACE-A, 2008-08-01 (a,b), and 2017-05-01 (c,d). Figures on the left and on the right show the same data, plotted using line and dot style for better visual demonstration.

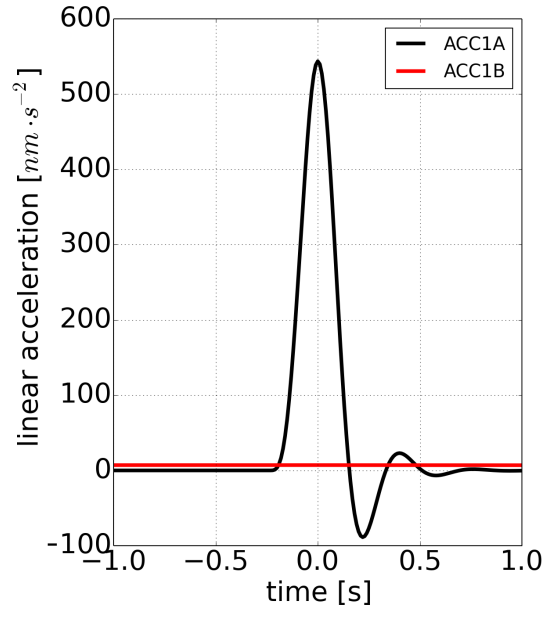

(a)

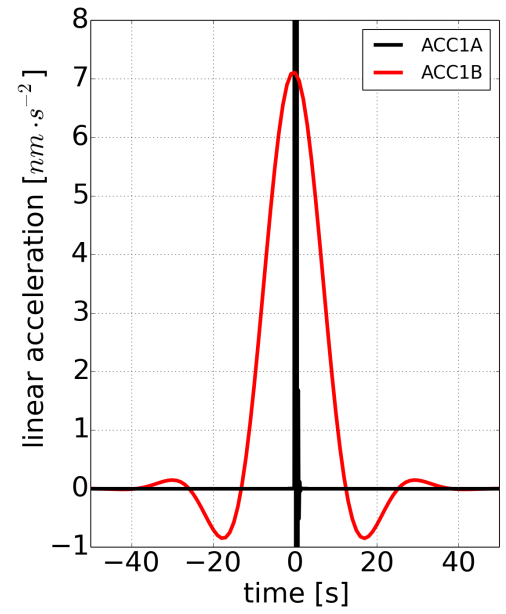

(b)

Figure 8: Due to their asymmetric shape, the thruster spikes remain in the ACC data even after $35 \mathrm{mHz}$ low-pass filtering. The original thruster spike is about $1 \mathrm{~s}$ long, but after $35 \mathrm{mHz}$ low-pass filtering it is smeared over approx. $70 \mathrm{~s}$. Note that both figures show the same spikes. The left figure zooms into the time axis, the right figure zooms into the ACC-axis

\subsection{Twangs}

Twangs are high-frequency, damped oscillations lasting for a few seconds. They dominate the radial component of the linear acceleration. The most likely cause of the twangs is vibration of insulating foil covering the bottom of each spacecraft (Frommknecht, 2008). An extended study on twangs was presented by Peterseim (2014), in which also other possible causes of these disturbances were discussed. 
Despite their large magnitude, up to a few thousand $n m \cdot s^{-2}$, and frequent occurrence, up to hundreds per day, their effect on the gravity field is negligible. Due to their symmetrical shape, the residual acceleration after the $35 \mathrm{mHz}$ low-pass filtering, is at or below the measurement accuracy. Therefore, the twangs do not affect the transplanted data and no additional correction for twangs is needed.

\subsection{Other disturbances}

In the linear acceleration, other disturbing signals can be found, such as heater switching spikes (Flury et al. 2008) or magnetic torquer induced spikes (Peterseim et al., 2012).

The heater switching spikes are caused by switching the heaters, which maintain the desired temperature of the spacecraft. However, the thermal control on both spacecraft was turned off in April 2011, thus the heater switching spikes are no longer present in the data. Since, for our analysis, we use data from 2015 to 2017, no correction for these spikes is necessary.

The magnetic torquer induced spikes are very small, just at or below the level of ACC measurement accuracy. These disturbances do not affect the gravity field as they are eliminated by the low-pass filter. Therefore they do not have to be corrected.

The off-centering of the ACC proof-mass with respect to the satellite's center of mass could cause additional signal in the linear acceleration due to satellite's angular motion and gravity gradients. To minimize this effect, center of mass calibration is regularly performed (Wang et al., 2010). Therefore we do not have to correct for this effect in the post-processing. 


\section{ACC data transplant}

The ACC data transplant uses GRACE-A ACC measurement to generate the missing GRACE-B ACC data. ACC data transplant is possible due to the fact that both satellites are flying in almost the same orbit, separated by approx. $220 \mathrm{~km}$. This means, the following satellite flies through nearly the same inertial position approx. 25 seconds later than the leading satellite. Within this time frame, it can be assumed that the change in the atmospheric density, solar radiation or Earth albedo is very little. Hence it can be assumed that the non-gravitational acceleration at that particular point is approximately the same for both spacecraft.

There are two different realizations of the ACC data transplant. We call them: the "simple" and the "full" ACC data transplant. The "simple" ACC data transplant includes only time and attitude correction (Save et al., 2006). The time correction resolves the orbit separation, and the attitude correction deals with the different attitude of each spacecraft relative to the velocity vector. The "full" ACC data transplant includes not only the time and the attitude correction, but also thruster spike correction, which represents the effect of the residual linear acceleration caused by thruster firings. Figure 9 shows a flowchart of individual processing steps for both ACC data transplant realizations. Table 2 provides a brief description of the GRACE data products used for ACC data transplant.

Because the PWM was turned off in Dec 2014, for thruster spike modeling we only use data from 2015 to 2017 in order to keep the data set consistent.

In the following, details about the time, attitude and thruster spike correction are provided. The numerical comparison of the "simple" and the "full" ACC data transplant is presented in Sections 4 and 5 .

\section{"simple" ACC data transplant}

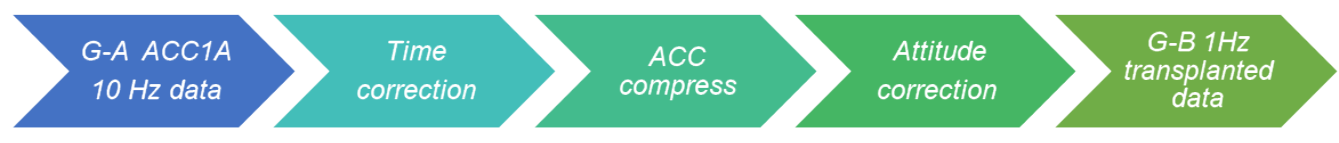

\section{"full" ACC data transplant}

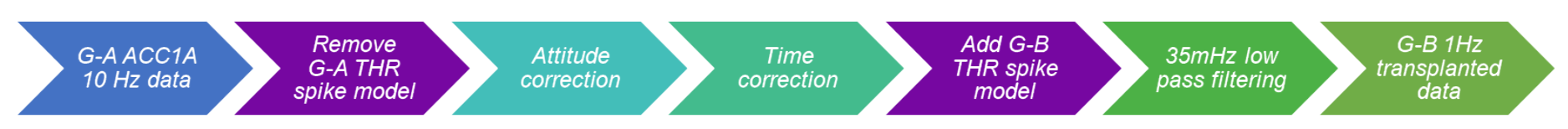

Figure 9: Flowchart of the key processing steps of the "simple" and "full" ACC data transplant

$\begin{array}{ll}\text { ACC1A } & \text { raw } 10 \mathrm{~Hz} \text { linear acceleration in AF and receiver clock time } \\ \text { ACC1B } & \text { 1s linear acceleration after } 35 \mathrm{mHz} \text { low-pass filtering, given in SRF and GPS time } \\ \text { CLK1B } & \text { offset of the satellite receiver clock time relative to GPS time } \\ \text { ECI1A } & \text { spacecraft orbits in inertial coordinates } \\ \text { THR1A } & \text { thruster activations and firing duration given in receiver clock time } \\ \text { THR1B } & \text { thruster activations and firing duration given in GPS time }\end{array}$

Table 2: GRACE Level-1 data products used in this study. A full data product description can be found in Bettadpur (2012)

\subsection{Time correction}

The time correction resolves the orbit separation. The core idea of the computation of the timeshift is finding the minimal 3D distance between the two spacecraft. The input data for the time correction are ECI1A and CLK1B data. As the satellites fly with a speed of approx. $7 \mathrm{~km} / \mathrm{s}$, the time correction has to be determined on sub-milisecond accuracy level. For this reason, the orbits are interpolated using Lagrange quadratic interpolation and the CLK1B clock correction is interpolated using cubic splines. Figure 10 shows the time correction due to orbit separation for one selected day. 


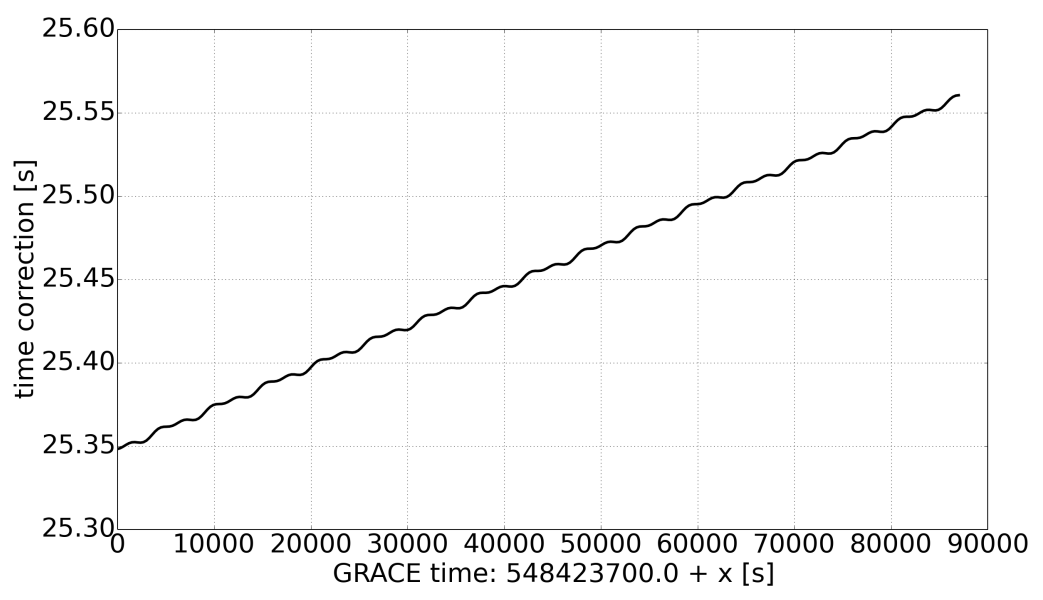

Figure 10: Time correction due to the orbit separation as on May 19, 2017

\subsection{Attitude correction}

The different orientation of the two spacecraft relative to the velocity vector is inevitable due to the precise inter-satellite pointing, which is one of the fundamental requirements of the GRACE missions. As the two satellites fly with their K-band antenna horns orientated towards each other, the leading satellite is rotated about $180^{\circ}$ about the radial axis and both satellites fly with a pitch offset of approx. $\pm 1^{\circ}$ with respect to the velocity vector, cf. Figure 12. Therefore, in order to rotate the ACC data from GRACE-A into GRACE-B reference frame, pitch and yaw rotations need to be applied, cf. Equation 1. Here, AF denotes the ACC reference frame, $\psi$ and $\phi$ denote yaw and pitch rotation angles, resp.

$$
\left[\begin{array}{l}
a_{x} \\
a_{y} \\
a_{z}
\end{array}\right]_{\mathbf{A F}^{*}}=\mathbf{R}_{\mathbf{y}}(\psi) \cdot \mathbf{R}_{\mathbf{x}}(\phi) \cdot\left[\begin{array}{c}
a_{x} \\
a_{y} \\
a_{z}
\end{array}\right]_{\mathbf{A F}}
$$

where

$$
\begin{aligned}
& \mathbf{R}_{\mathbf{x}}(\phi)=\left[\begin{array}{ccc}
1 & 0 & 0 \\
0 & \cos \phi & \sin \phi \\
0 & -\sin \phi & \cos \phi
\end{array}\right] \\
& \mathbf{R}_{\mathbf{y}}(\psi)=\left[\begin{array}{ccc}
\cos \psi & 0 & -\sin \psi \\
0 & 1 & 0 \\
\sin \psi & 0 & \cos \psi
\end{array}\right]
\end{aligned}
$$

Figure 13 shows the pitch offset of each spacecraft relative to the velocity vector for November 2016 to June 2017. Until end of March 2017, the satellites were kept precisely pointed toward each other, cf. Figure 12a, which resulted in a non-zero pitch offset relative to the velocity vector. The magnitude of the pitch offset varied between $\pm 0.6^{\circ}$ and $\pm 1.3^{\circ}$. Our empirical analysis based on data from 2015-2016 revealed that the best fit of the rotated GRACE-A data and the original GRACE-B data is achieved when applying $3.2^{\circ}$ pitch correction instead of the actual angle, which would be between $1.2^{\circ}$ and $2.3^{\circ}$. Also, the analysis of the computed gravity fields based on the different pitch settings shows that the gravity field solution is best when using the $3.2^{\circ}$ pitch correction, cf. Figure 11. One possible explanation for that could be that this value accounts not only for the true pitch offset, but also for differences in rarefied airflow around the spacecraft. 


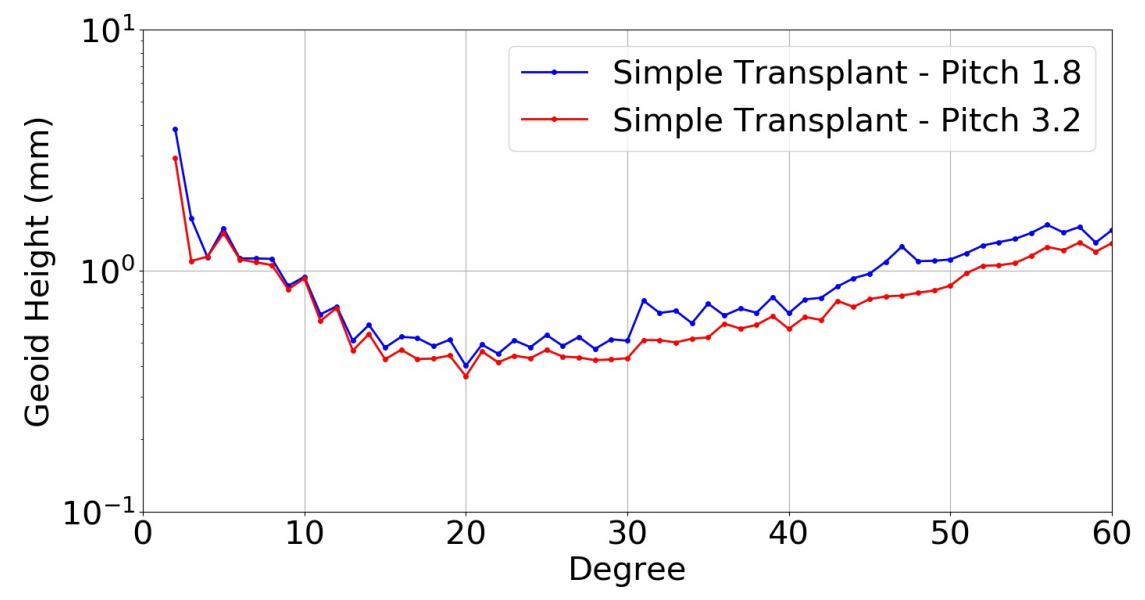

Figure 11: RMS of degree variances of monthly gravity field solutions for April 2015 to November 2016, obtained by "simple" ACC transplant while applying $1.8^{\circ}$ and $3.2^{\circ}$ pitch correction, respectively.

On March 29, 2017, the pitch offset of both spacecraft was intentionally changed in order to align the $x_{S R F}$ axis with the velocity vector, cf. Figure $12 \mathrm{~b}$. The decision to change the satellite orientation was made to support the ACC data transplant. After the final attitude correction commanded on April 7, 2017, the pitch offset of both spacecraft was zero, see Figure 13 . This means, only the yaw correction is needed to rotate the ACC data after this date.

The change of the pitch offset, however, introduced a large bias of $\approx 15 \mathrm{mrad}$ in the pitch pointing angle with respect to the line-of-sight. This misalignment acts as an amplification factor on the attitude noise. In addition, the attitude noise further increased during single star camera operation, i.e. when the other camera was blinded by the Sun or the Moon. Consequently, zonal coefficients of the spherical harmonics of the gravity field solutions have been significantly affected. However, the new V03 of SCA1B data product, i.e. combined attitude solution based on star camera quaternions and accelerometer angular accelerations, helped to significantly reduce this noise and to mitigate the latitudinal banding in the gravity field solutions (Wiese et al., 2017; Sakumura et al. 2017).

Note that for the purpose of the ACC data transplant, we only account for the $180^{\circ}$ yaw rotation and the $3.2^{\circ}$ or $0^{\circ}$ pitch offset. We do not correct for the actual attitude variations measured by the onboard star cameras. The star camera measurement has much higher high-frequency noise than the accelerometer measurement (Frommknecht, 2008). Therefore if using the star camera data for the attitude correction, additional high-frequency noise would be introduced to the transplanted ACC data, which is not desirable. 


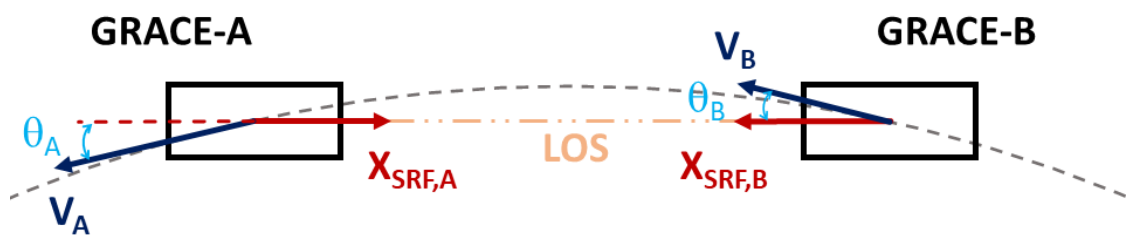

(a) non-zero pitch offset

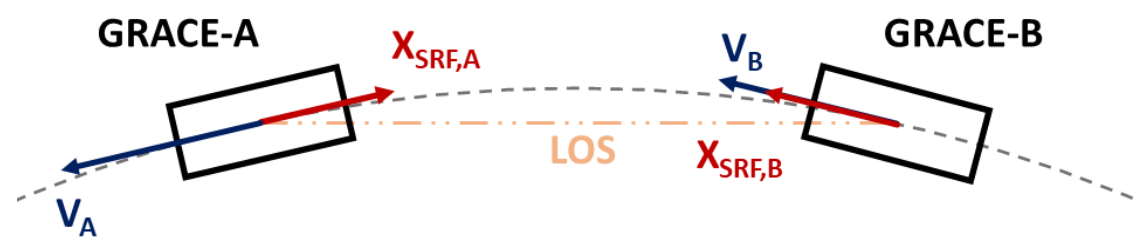

(b) zero pitch offset

Figure 12: Sketch of the satellite orientation with respect to the velocity vector and the line-of-sight (LOS) during the science mode operation. Case a): until end of March 2017, the satellites were kept precisely pointed towards each other, which resulted in a non-zero $\left(\approx \pm 1^{\circ}\right)$ pitch offset. Case b): at the end of March 2017 , the satellite's pointing with respect to the velocity vector was intentionally changed to minimize the pitch offset, which resulted in attitude bias with respect to the line-of-sight

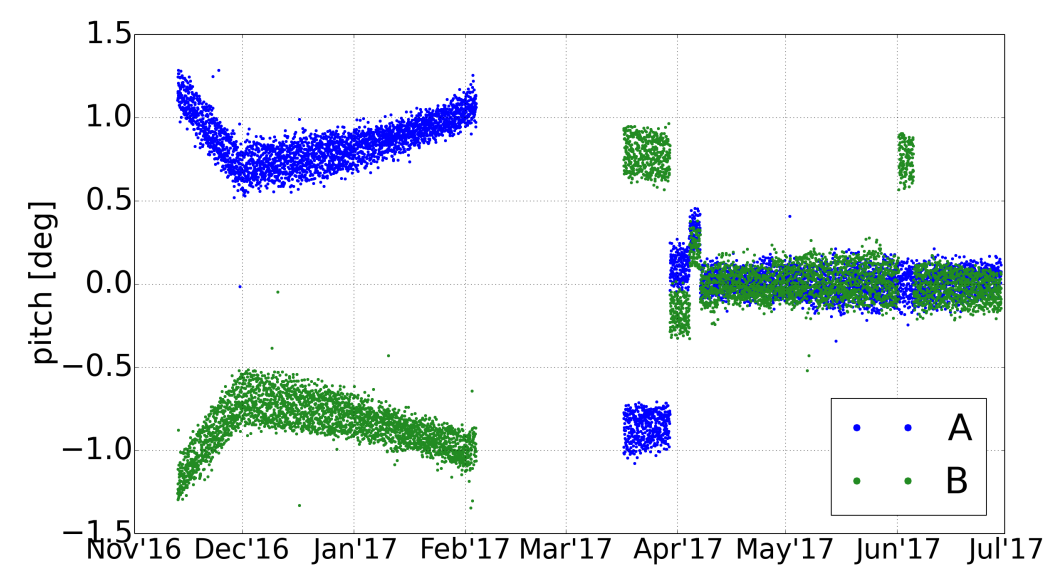

Figure 13: Timeseries of pitch offset angle with respect to the velocity vector for both GRACE-A and GRACE-B, from November 2016 to June 2017. Data from February to mid March 2017 was discarded because of the ongoing satellite swap maneuver

\subsection{Thruster spike correction}

The thruster spike modeling is the most challenging part of the ACC data transplant. It requires extensive data analysis and deep understanding of the ACC measurement system. In the following, we provide details about the new improved thruster spike model and also discuss its limitations. 


\subsubsection{Thruster impulse response function}

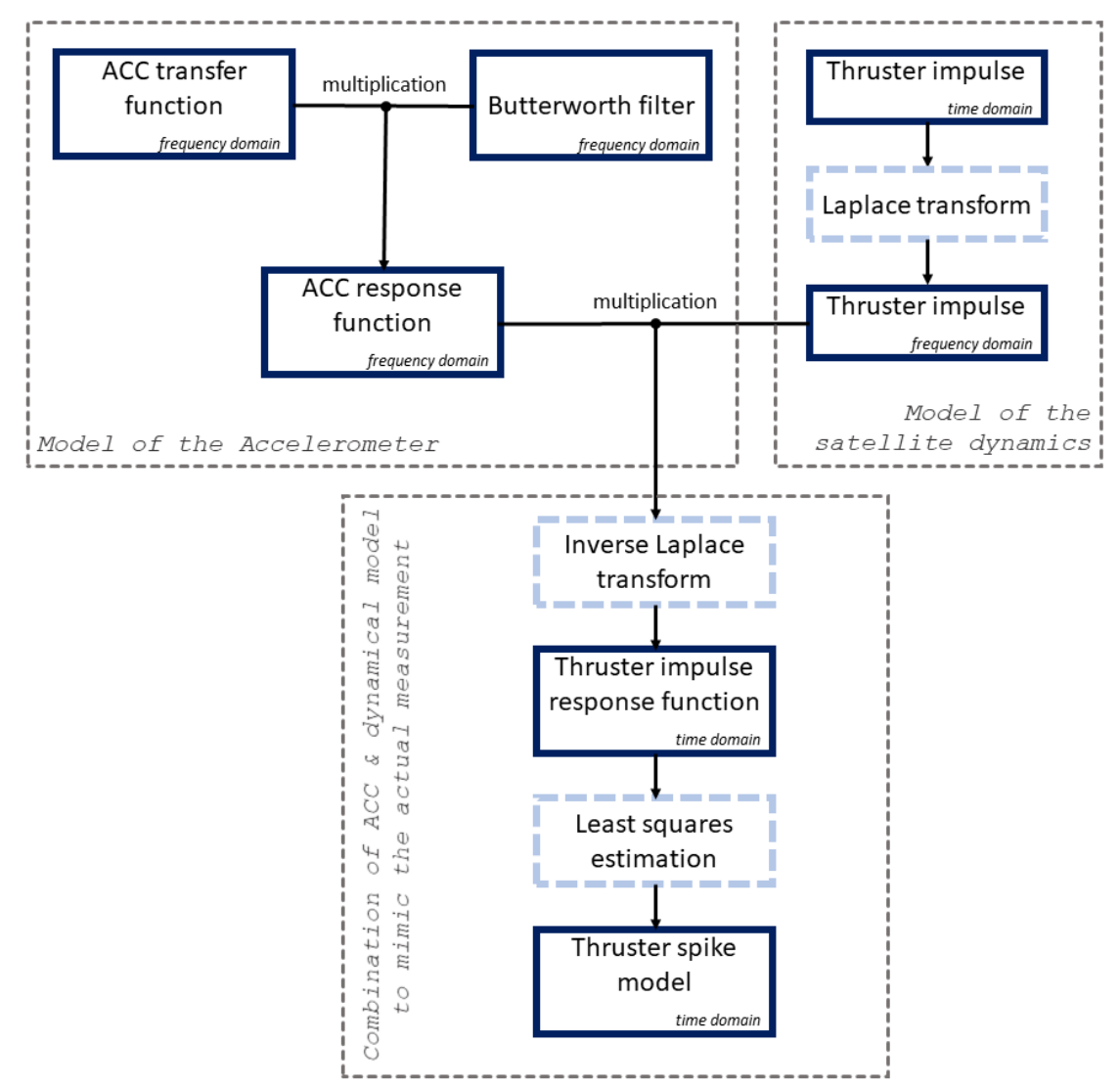

Figure 14: Flowchart of derivation of the thruster spike model

The thruster spike model is an analytic function determined based on inverse Laplace transform of the accelerometer impulse response function. A flowchart of the derivation of the thruster spike model is shown in Figure 14.

In the first step, we derived the ACC response function. This is done in frequency domain by multiplication of ACC transfer function (Equation 4) and Butterworth filter. The ACC transfer function is a mathematical function given by the manufacturer (ONERA), which describes a response of the system to the input signal. This function differs for the ultra-sensitive and the less-sensitive axes. The Butterworth filter is a $4^{\text {th }}$ order low-pass filter with cut-off frequency of $3 \mathrm{~Hz}$, which is applied onboard the spacecraft to de-alias the raw data. For more details, see Section 3.3 .2 .

In the second step, the thruster impulse was transformed from time domain into frequency domain using a Laplace transform. Based on an empirical data analysis (cf. Figure 22), we chose to model the thruster impulse in cross-track and radial direction as a single square pulse, and the thruster impulse in along-track direction as a two pulse function - ramp shaped pulse followed by a square pulse, see Figure 15. 


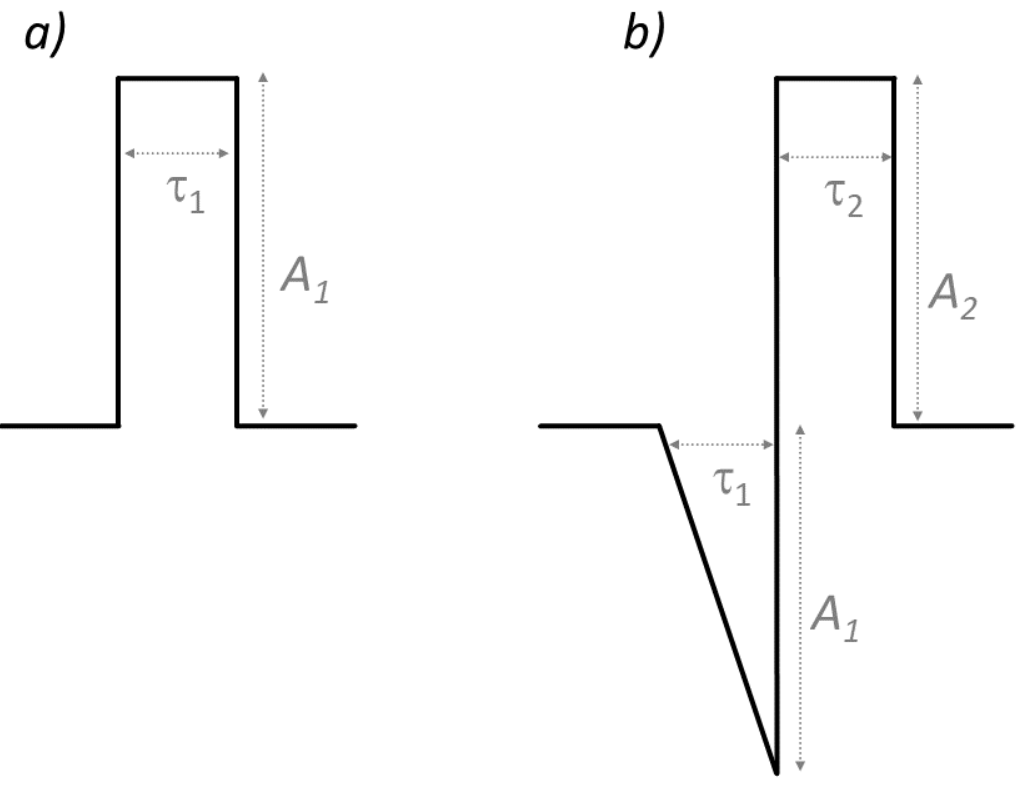

Figure 15: A sketch of the thruster impulse models: a) single square pulse used to model the impulse cross-track ( $\mathbf{x}_{\mathbf{A F}}$ ) and radial ( $\left.\mathbf{y}_{\mathbf{A F}}\right)$ direction, b) double ramp/square pulse used to model the impulse in along-track $\left(\mathbf{z}_{\mathrm{AF}}\right)$ direction . The shape of the pulse is driven by its width $\left(\tau_{1}, \tau_{2}\right)$ and amplitude $\left(A_{1}, A_{2}\right)$

Then, by multiplication of the ACC response function and the thruster impulse we obtained the thruster impulse response function in the frequency domain, which was further transformed into the time domain applying an inverse Laplace transform. Since the input function was very long and complicated, this step was done using Mathematica (Wolfram Research, Inc., 2016).

The output from Mathematica was converted to a function, which is driven by 5 parameters describing the input thruster impulse (cf. Figure 15):

$t_{0} \ldots$ timeshift $[s]$

$A_{1} \ldots$ amplitude of the first pulse $\left[\mathrm{m} / \mathrm{s}^{2}\right]$

$\tau_{1} \ldots$ width of the first pulse $[s]$

$A_{2} \ldots$ amplitude of the second pulse $\left[\mathrm{m} / \mathrm{s}^{2}\right]$

$\tau_{2} \ldots$ width of the second pulse $[s]$

These three and five variables, resp., were estimated by finding the best fit of the thruster impulse response function to the actually measured thruster spikes using the method of least squares. The model variables are estimated for all possible thruster spikes, i.e. 6 thruster pairs, firing duration from $30 \mathrm{~ms}$ to $1000 \mathrm{~ms}$, XYZ component linear acceleration and both satellites. Because the problem is highly non-linear, a clean set of ACC1A thruster spikes without outliers, as well as precise apriori values of the 5 variables are necessary.

\subsubsection{Accelerometer model}

GRACE SuperSTAR accelerometer is based on the electrostatic levitation of the proof-mass, with almost no mechanical contact with the cage. The proof-mass is controlled on its six degrees of freedom ( 3 translations and 3 rotations) through 6 pairs of electrodes, which apply the electrostatic forces. Due to the geometrical configuration of the proof-mass and the need to levitate it on ground under 1g, there are ultra-sensitive electrodes, which control horizontal translations along $\mathbf{y}_{\mathbf{A F}}$ and $\mathbf{z}_{\mathbf{A F}}$ axes and rotation about $\mathbf{x}_{\mathbf{A F}}$ axis, and less-sensitive electrodes, which control translation along $\mathbf{x}_{\mathbf{A F}}$ axis and rotations about $\mathbf{y}_{\mathbf{A F}}$ and $\mathbf{z}_{\mathbf{A F}}$ axes.

The pair of electrodes corresponding to each loop is used for both capacitive position sensing and electrostatic restoring force generation. Figure 16 shows the control loop for the sensitive axes of the accelerometer. The motion of the ACC proof-mass is detected by capacitive detectors, there is one detector for each electrode pair. For each degree of freedom, there is a Proportional-Integral-Derivative (PID) type controller, which delivers voltages in order to nullify its inputs. These voltages are sent to the measurement chain (data sent to ground after anti-aliasing filtering and decimation), as well as to the Drive Voltage Amplifiers (DVA) to be applied on the 
electrodes pairs. The proof-mass is supplied by DC voltage $V_{p}$ to linearize the electrostatic forces and a $100 \mathrm{kHz}$ voltage $V_{d}$ for the capacitive detection.

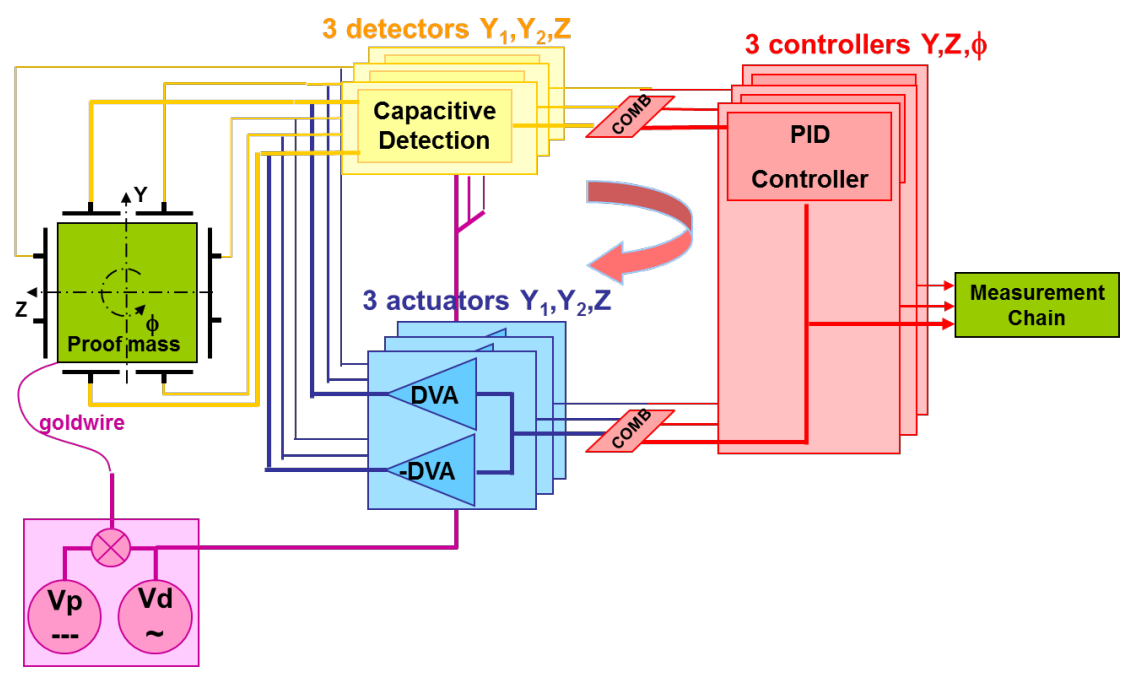

Figure 16: Schematic diagram of the accelerometer control loops for the sensitive axes, controlling 3 degrees of freedom - translation along the $\mathbf{y}_{\mathbf{A F}}$ and $\mathbf{z}_{\mathbf{A F}}$ axes and rotation $\phi$ about the $\mathbf{x}_{\mathbf{A F}}$ axis

The mathematical formulation of the electrostatic forces applied on the proof-mass as well as the measurement of each electronic transfer function allows to compute the accelerometer loop transfer function $H(p)$ (Touboul et al., 1999a):

$$
H(p)=\frac{\omega_{c}^{2}\left(1+b \cdot p+c \cdot p^{-1}\right)}{p^{2}+\omega_{c}^{2}\left(1+b \cdot p+c \cdot p^{-1}\right)-\omega_{p}^{2}}
$$

where

$$
\begin{aligned}
& p=i 2 \pi f \text { where } f \text { is frequency } \\
& \omega_{c}^{2} \text { is the accelerometer loop gain } \\
& \omega_{p}^{2} \text { is due to the electrostatic stiffness } \\
& b \text { is the derivative gain of the PID } \\
& c \text { is the integral gain of the PID } \\
& \ldots \text { for } \omega_{c}, \omega_{p}, b, c \text { values see Table } 3
\end{aligned}
$$

Table 3: Parameters of the accelerometer loop transfer function (Equation 4 for each linear acceleration in Nominal Range Mode (NRM), used for scientific sessions

\begin{tabular}{cccc}
\hline & $\mathbf{x}_{\mathbf{A F}}$ & $\mathbf{y}_{\mathbf{A F}}$ & $\mathbf{z}_{\mathbf{A F}}$ \\
$\omega_{c}[\mathrm{rad} / \mathrm{s}]$ & 74 & 10.4 & 10.4 \\
$\omega_{p}[\mathrm{rad} / \mathrm{s}]$ & 7.6 & 1 & 1 \\
$b[\mathrm{~s} / \mathrm{rad}]$ & $3.6 \cdot 10^{-2}$ & 0.2 & 0.2 \\
$c[\mathrm{rad} / \mathrm{s}]$ & 4.1 & 0.9 & 0.9 \\
\hline
\end{tabular}

This transfer function can be verified on ground for the ultra-sensitive axes $\mathbf{y}_{\mathbf{A F}}$ and $\mathbf{z}_{\mathbf{A F}}$ (Figure 17). The accuracy of the verification is limited by the seismic noise. The model is representative of the true accelerometer transfer function up to frequency of $2-3 \mathrm{~Hz}$. It is due to the fact that the model doesn't take into account the detector and DVA transfer function (just the gain) as well as the low-pass filter at the output of the controller. It is sufficient as this transfer function will be multiplied by the 4th order Butterworth anti-aliasing filter. For the less sensitive axis $\mathbf{x}_{\mathbf{A F}}$, the transfer function determination is based only on individual measurement of each 
electronic transfer function as well as the control of geometrical parameters (like the gap between proof-mass and electrodes and the proof-mass mass).

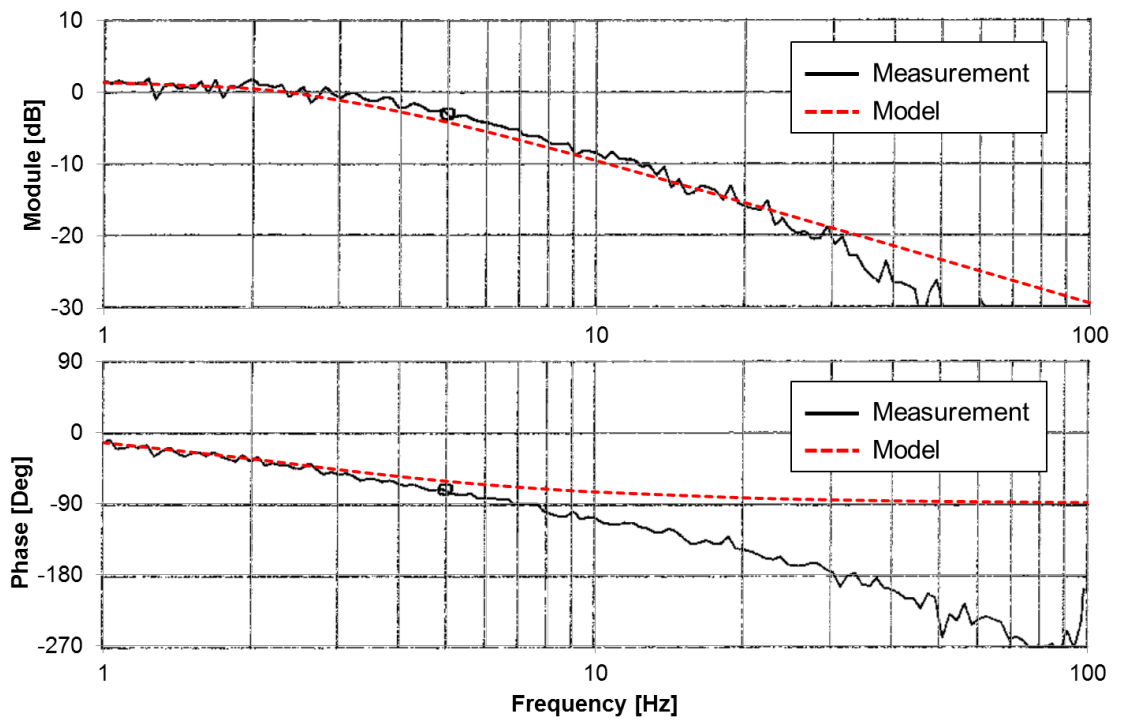

Figure 17: On-ground verification of the transfer function for GRACE SuperSTAR FM2 (flight model 2) accelerometer, for yAF axis in Nominal Range Mode (NRM). The upper plot shows the module of the transfer function in dB and the lower plot shows the phase of the transfer function in degrees. The red dotted curve is the expected transfer function response according to the model and the black curve is the measurement done on ground

The measurement chain is composed by the pick-off filter, a 1st order low pass filter with a cut-off frequency higher than $80 \mathrm{~Hz}$, the anti-aliasing filter, a 4th order Butterworth low pass filter with a -3dB cut-off frequency at $3 \mathrm{~Hz}$ and a gain of 5 and the sigma-delta analog to digital converter with a order 3 sinc transfer function with a $-3 \mathrm{~dB}$ cut-off frequency at $2.619 \mathrm{~Hz}$. Only the 4th order Butteworth low-pass filter was taken into account in the accelerometer response function.

\subsubsection{Thruster spike model}

The measured thruster spikes are extracted from the raw $10 \mathrm{~Hz}$ ACC1A timeseries and thruster spikes of the same type are then clustered together. The thrusters are activated either individually or in a sequence, where multiple pairs can be activated at the same time. Therefore, in order to get a representative thruster spike data set, the extracted spikes have to pass the following criteria: at the time of thruster activation, only one thruster pair can be activated and within $3 \mathrm{~s}$ before and after the thruster activation, no other thruster firing can occur. In the next step, thruster spikes, which are superimposed by twangs, are discarded from the data cluster.

Figure 18 shows a clean data set of + roll $100 \mathrm{~ms}$ thruster spikes and the fitted model function. Our mathematical model replicates the shape of the real spike very well.

For the most frequent thruster events (see Table 1), it is possible to obtain a timeseries of the estimated parameters. Figure 19 shows daily estimates of the model parameters for + roll $100 \mathrm{~ms}$ thruster spike in 2016. Clearly, the parameter values are consistent in time. This is an important finding, because it allows us to use data from multiple years to get clusters of measured thrusters spikes for the less frequent thruster events. Also, it allows us to create a thruster spike model for GRACE-B which is based on data from 2015 to Sep 2016, while this model is used to correct data from Nov 2016 to Jun 2017. 


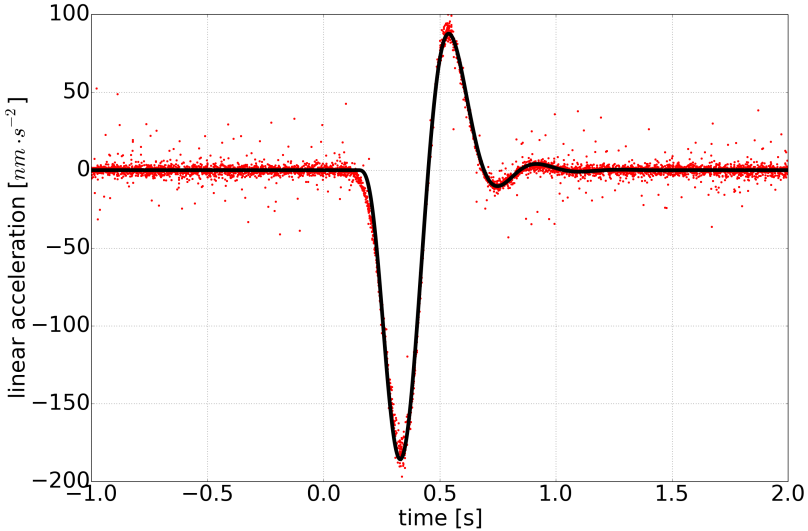

(a) along-track

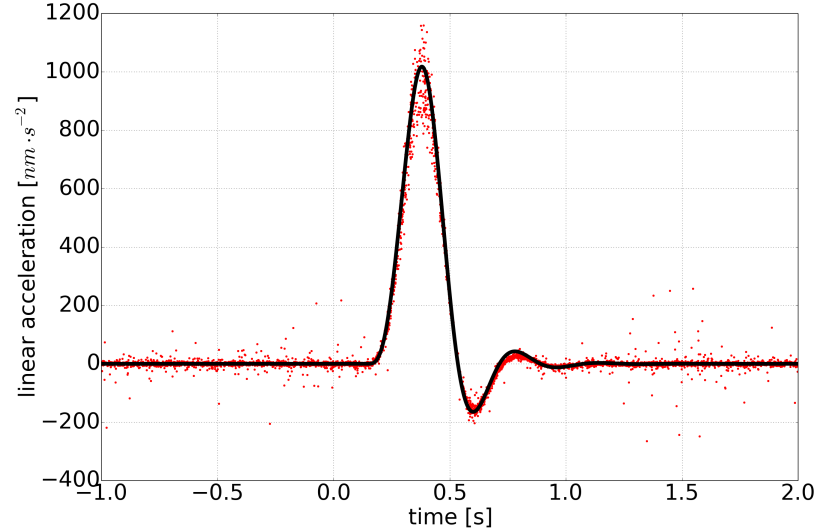

(b) cross-track

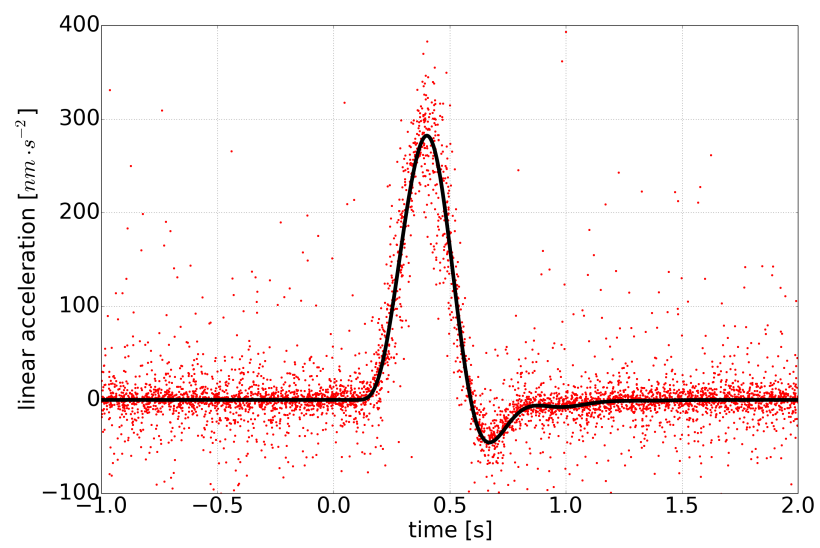

(c) radial

Figure 18: Fit of the thruster spike model (black curve) to the measured thruster spikes (red dots) induced by + roll $100 \mathrm{~ms}$ thrust on GRACE-A. The red dots represent approx. 180 thruster spikes (extracted from ACC1A data) 

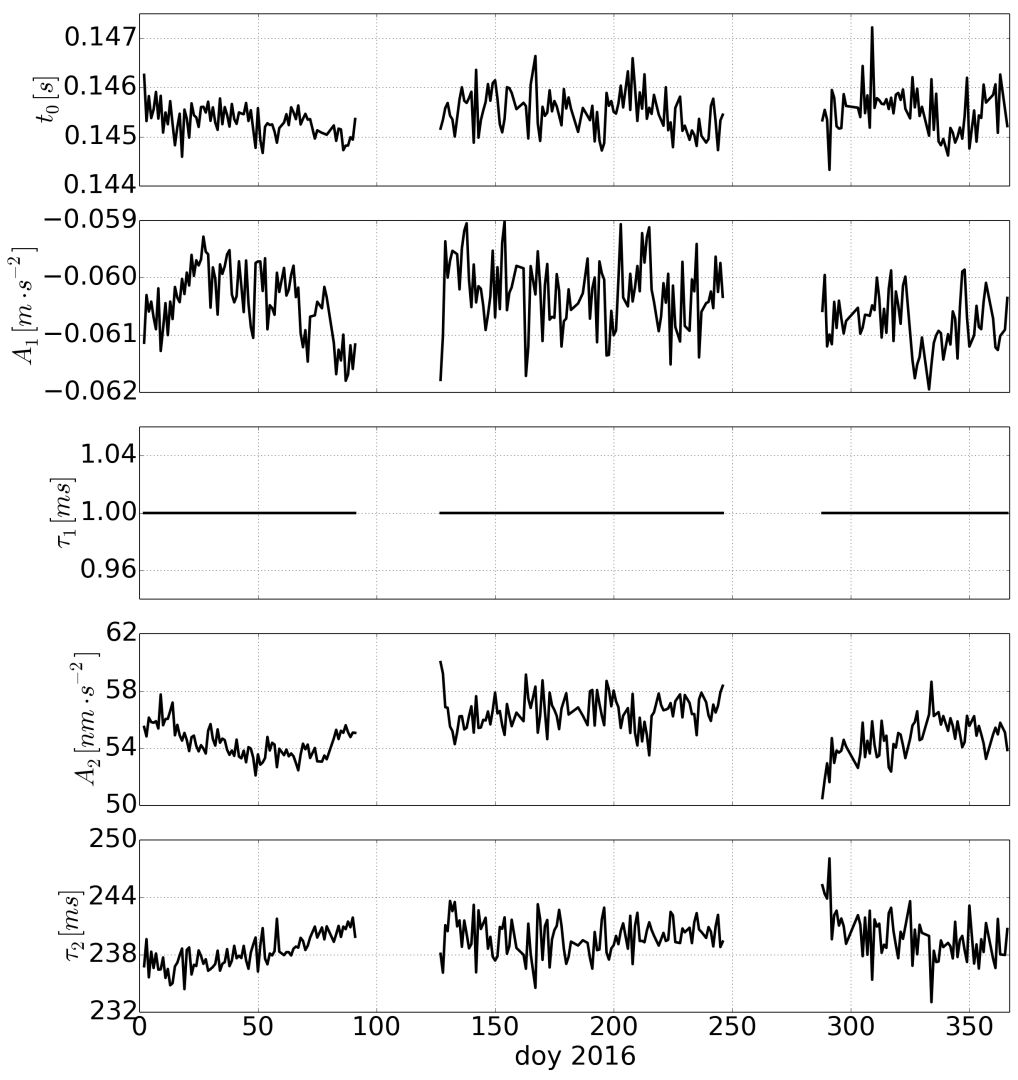

Figure 19: Daily estimated values of thruster model parameters for GRACE-A + roll 100 ms events, along-track component, for 2016. Note that $\tau_{1}$ is set to a constant value to stabilize the solution

The parameter values can be directly estimated for thruster spike types, for which a clean data set of ACC1A data was available. This, however, did not cover the full range of the possible thruster firing durations. The reason for that is that the thruster events with long duration are rare and they occur in sequences, hence it is not possible to separate the spikes from each other and get a clean data set. Therefore, in order to get the thruster spike model for any arbitrary firing duration, the timeseries of the directly estimated model parameters had to be interpolated and extrapolated. As Figure 20 shows, the parameter estimates for the various firing duration are very consistent, which allows to find an interpolation curve for each parameter. The respective thruster spike models for the different firing duration based on these interpolated data are shown in Figure 21. 

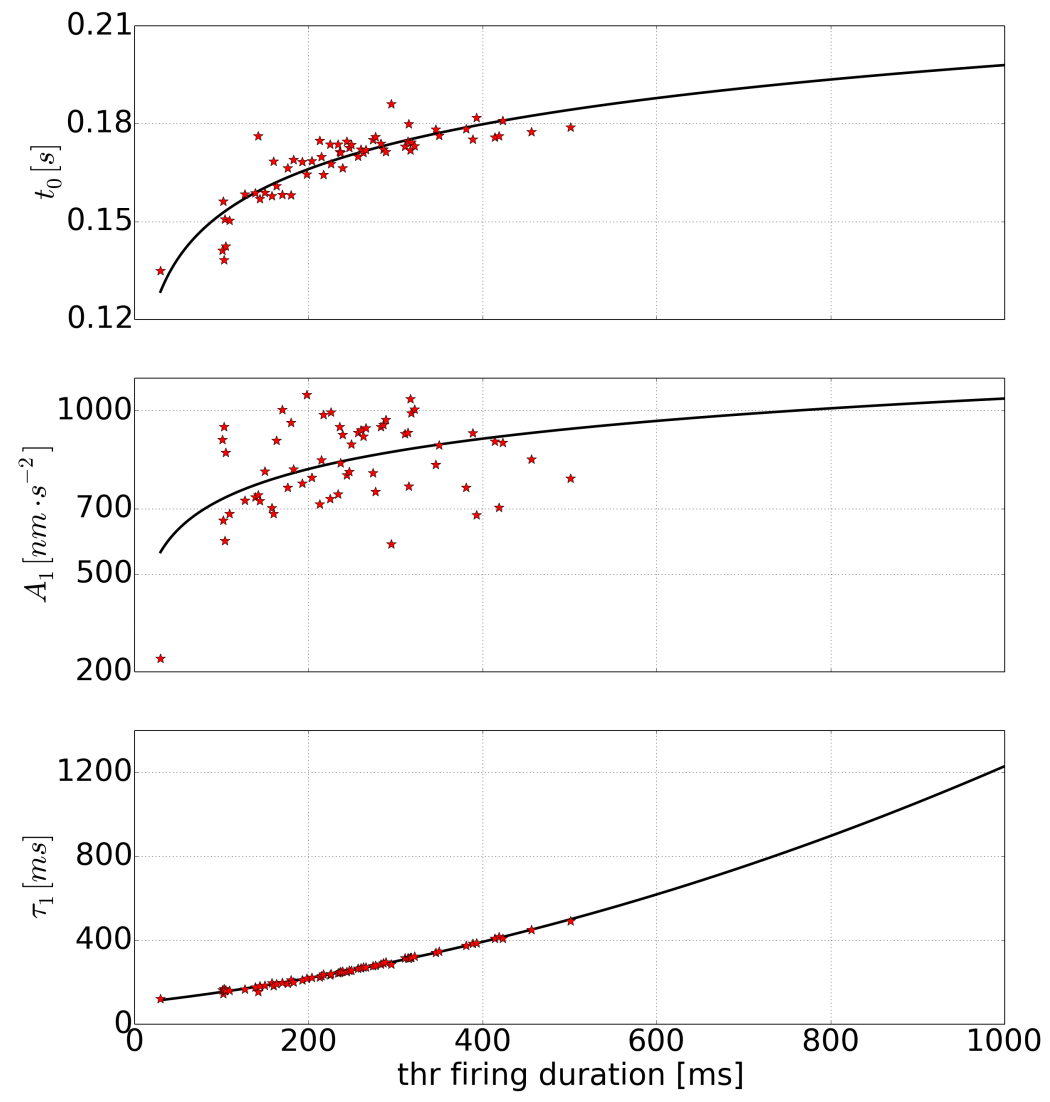

Figure 20: Thruster spike model parameters for GRACE-A + roll thruster event, cross-track component, for the whole range of firing duration. The red stars represent values which were directly estimated based on available ACC1A data. The black curves represent the interpolated solution for each parameter

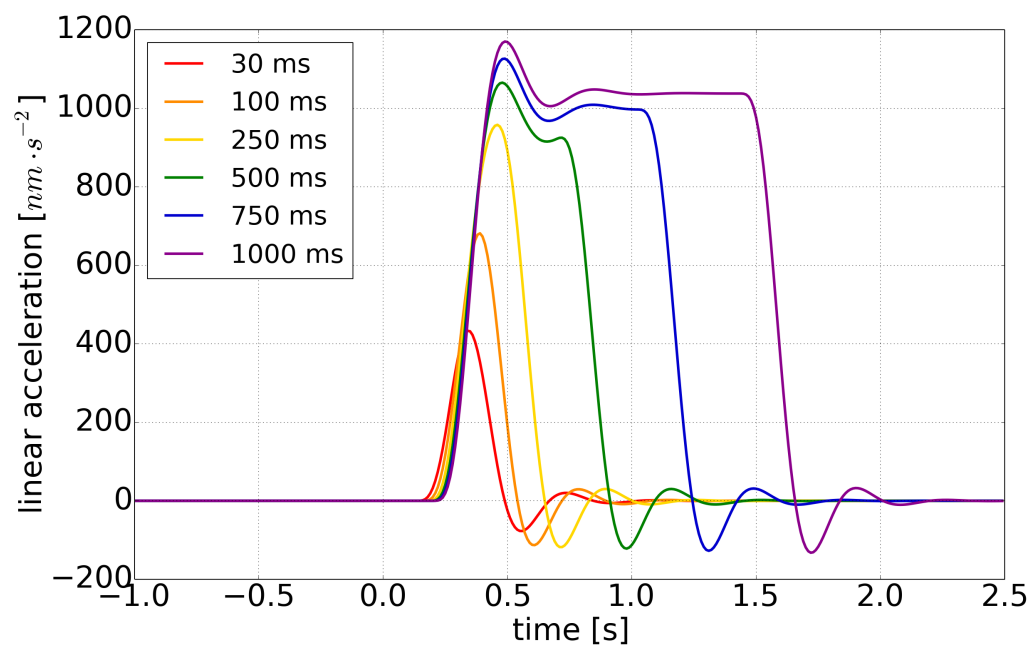

Figure 21: Thruster spike models for GRACE-A + roll thruster pair, for firing duration between $30 \mathrm{~ms}$ and $1000 \mathrm{~ms}$, cross-track component of the linear acceleration

While the thruster spike model based on a single square pulse provides a very good fit to the measured spikes in radial and cross-track component, the fit to the measured data in along-track component is poor. For comparison, Figure 22 shows the fit of two thruster spike models to the along-track thruster spikes. The two models differ in the used function for the thruster impulse, which is a single square pulse in the first case and a double ramp/square pulse in the second case. The second model provides four times better fit then the first 
one. The RMS of the differences between the respective model and the measured data for the given example is $24 \mathrm{~nm} \cdot \mathrm{s}^{-2}$ and $6.6 \mathrm{~nm} \cdot \mathrm{s}^{-2}$, resp.

Note that for both spacecraft the shape of all along-track thruster spikes is consistent for all thruster pairs, only its magnitude differs, cf. Figure $5 \mathrm{a}$ and $5 \mathrm{~b}$. We can confirm that the shape and the magnitude of the individual thruster spikes are independent of spacecraft's attitude, hence they are not driven by factors such as air drag. Our hypothesis is that the shape of the along-track thruster spike is driven by two factors: thruster misbalance itself and mechanical design of the spacecraft's propulsion system. That would also explain why the second model (i.e. based on thruster impulse modeled as a double ramp/square pulse) provides significantly better fit to the measured data.

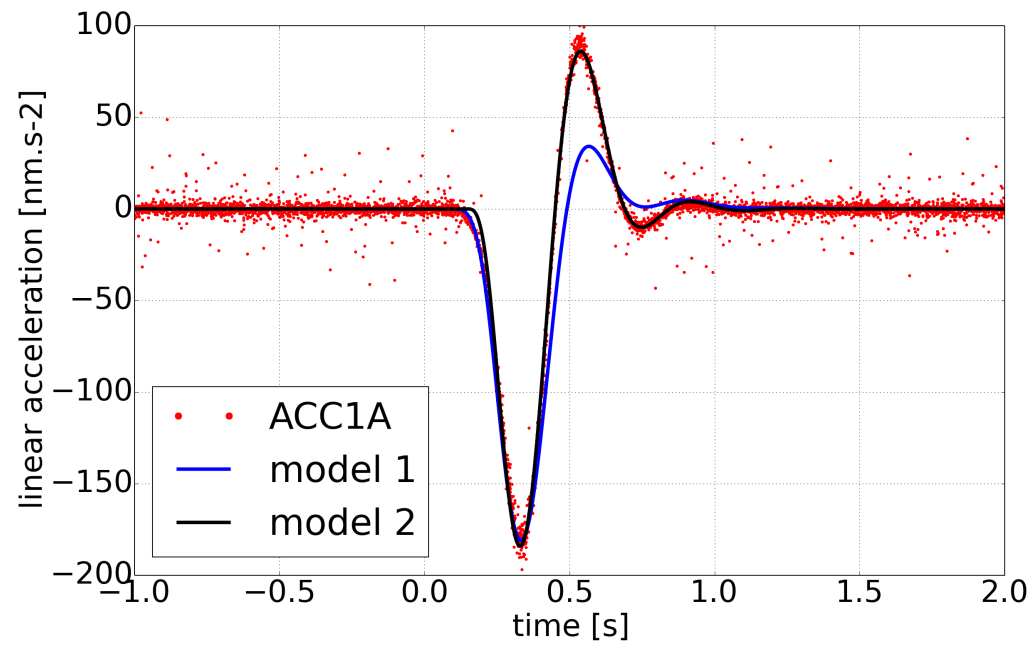

Figure 22: Comparison of the fit of two thruster spike models to the along-track thruster spikes (GRACE-A, +roll, 100 ms, extracted from ACC1A). Model 1 was derived using a single square pulse to model the thruster impulse. Model 2 was derived using a double ramp/square pulse

\subsubsection{Model limitations}

The accuracy of the thruster spike model is limited by several factors:

- least squares estimation: The estimated model parameters represent an "average" spike, cf. Figure 18 Despite the very good fit to the data cluster, residual acceleration may still remain in the ACC data after the thruster spike correction is applied.

- interpolation/extrapolation: Due to the lack of clean clusters of ACC1A thruster spikes, the interpolation/extrapolation of the estimated model parameters is inevitable, cf. Figure 20. It can be assumed that the spikes with shorter firing duration are estimated more precisely that the ones with long firing duration.

- ambiguity in THR data files: In principle, commands for thruster activation can be sent by the onboard Attitude and Orbit Control System (AOCS) every second. However, the onboard system provides only a cumulative information about thruster activations every three seconds. For this reason, when the thrusters were activated in a close sequence, it is not possible to distinguish which thruster pair was activated first and for how long. This cumulative information introduces a serious ambiguity which cannot be resolved.

- valve pressure change: The analysis of long timeseries of the estimated model parameters show that one of the factors affecting the amplitude of the thruster spikes may be thruster valve pressure. This became obvious when in March 2017, the valve pressure onboard GRACE-B was intentionally changed. Because the accelerometer on GRACE-B was turned back on in May 2017 for 20 days, it was possible to estimate directly the model parameters and compare them with the values in 2015 and 2016. Although the change in the amplitude of the thruster spikes was noticeable, we still used the same model for all months, which provided a satisfactory fit to the data. 
- ACC scale factors: The measured linear accelerations have to be corrected for bias and scale, which are unique for each accelerometer. This is done first during the gravity field processing. Our thruster spike model is based on ACC data before scaling. However, the introduced inaccuracy in the thruster spike correction is assumed to be very small, because the GRACE-A and GRACE-B scale factors are very similar.

Despite all these limitations, in Section 4 we show that applying the thruster spike correction based on our model results in significant improvement of the transplanted ACC data.

\section{Validation of the ACC data transplant}

ACC data transplant is validated by comparison of the transplanted data to the original GRACE-B ACC measurement for a selected period of time, May 2-22, 2017, when on both satellites the accelerometer was turned on. In the following, we present the comparison of the "simple" and "full" ACC data transplant with the original GRACE-B ACC measurement in terms on ACC timeseries as well as gravity field solution based on these three data sets.

\subsection{ACC timeseries}

Figure 23 shows a comparison of all three components of the Level-1B linear acceleration obtained by "simple" and "full" ACC data transplant, with the original GRACE-B ACC measurement. The only difference between the "simple" and the "full" ACC data transplant is the thruster spike correction. While in the first case the linear accelerations still contain GRACE-A thruster spikes, in the second case, these GRACE-A thruster spikes are removed from the data set and the GRACE-B thruster spikes are added back.

The linear acceleration generated by the "full" ACC data transplant is characterized by significantly reduced high frequency noise compared to the simple ACC data transplant. This is solely a result of precise thruster spike modeling and the respective thruster spike correction. For a better visibility, Figure 24 shows timeseries and power spectral densities of differences of the respective transplanted data and the original GRACE-B measurement. A major improvement is achieved in cross-track and radial component. Especially frequencies above $5 \cdot 10^{-4} \mathrm{~Hz}$ are affected.

A smaller effect in the along-track component is to be expected. One reason is that the thrusters are intentionally located off the along-track axis, therefore the residual linear acceleration due to thruster firing is the smallest of all components. Another and more important reason is that the noise in the along-track component is dominated by high-frequency variations in the Earth's atmosphere, which cannot be modeled.

Figures 24b, 24d and 24f. however, reveal a 1/rev signal remaining in the transplanted data. As the ACC measurement itself is dominated by $1 / \mathrm{rev}$ signature, it is not trivial to separate the true $1 / \mathrm{rev}$ signal and the $1 /$ rev error due to transplant. Correcting for this $1 /$ rev signal would require extensive data analysis and experiments based on true ACC measurements from both satellites for at least one half beta cycle (approx. 6 months). Possibly more beta cycles would be better so as not to derive models and statistics on a single beta cycle driven by varying solar activity, etc. In addition, towards the end of the mission (2016/2017), the satellites are very low in orbit. The transplant error, dominated by $1 / \mathrm{rev}$, at this altitude is an order of magnitude higher than earlier in the mission (2008-2010) because the error is signal proportional and the signal is an order of magnitude higher in some directions. Another factor is the change of angle of attack in March 2017, which would require to recalibrate the $1 / \mathrm{rev}$ model. This data analysis, however, is beyond the scope of this paper, and is a subject for future work. 


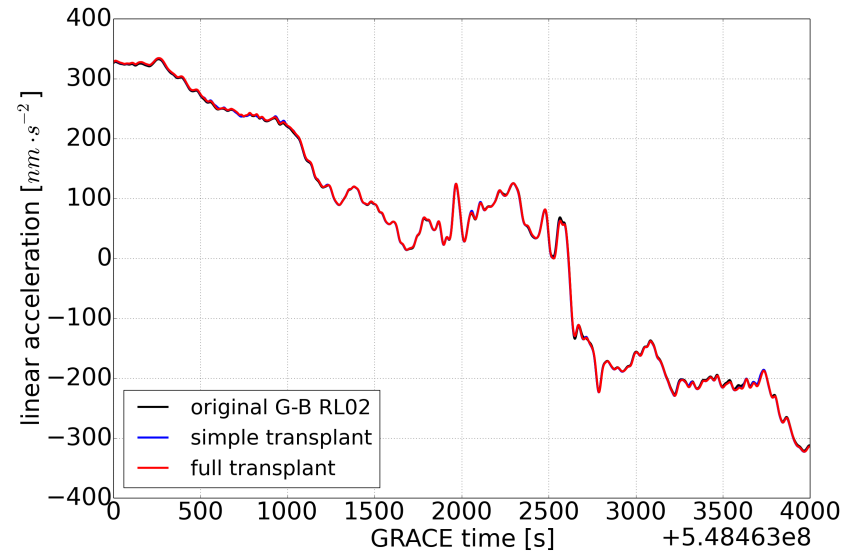

(a) along-track

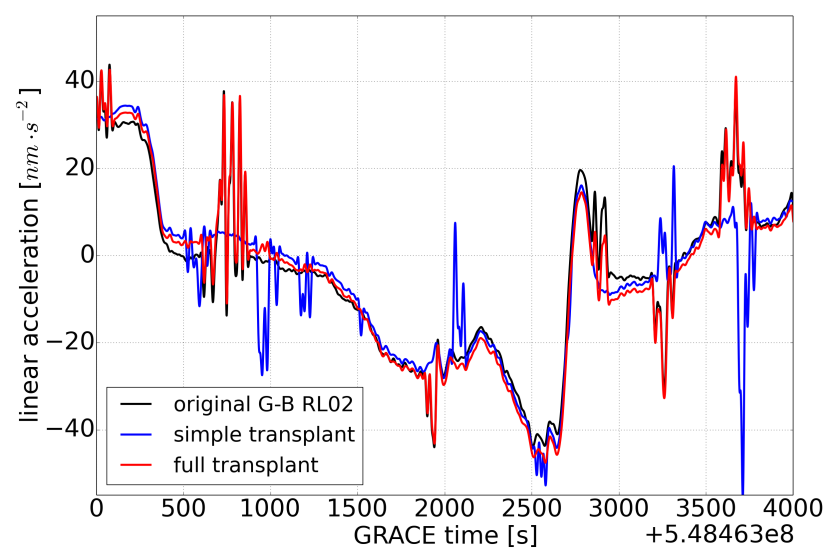

(b) cross-track

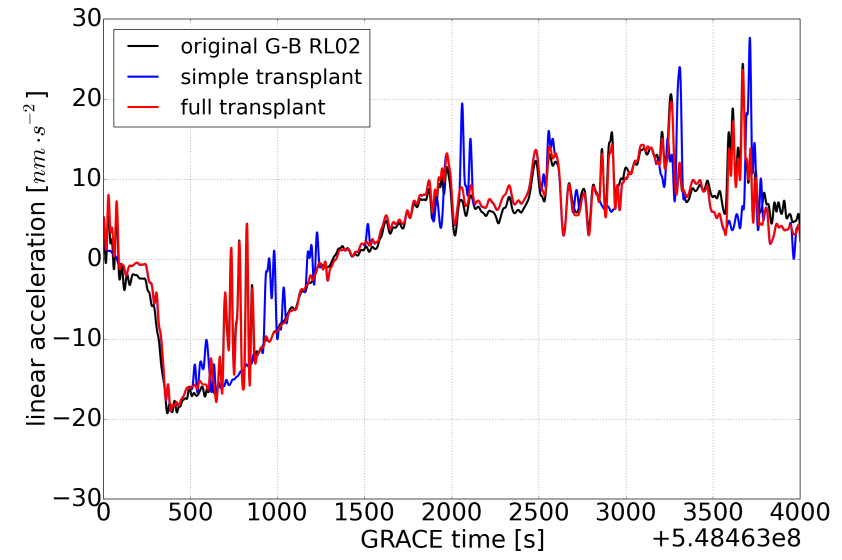

(c) radial

Figure 23: Comparison of the Level-1B (1 Hz, $35 \mathrm{mHz}$ low-pass filtered) linear acceleration obtained by "simple" (blue curve) and "full" (red curve) ACC data transplant with the original GRACE-B ACC data (black curve) as measured by the onboard accelerometer. Data sample from 2017-05-19 


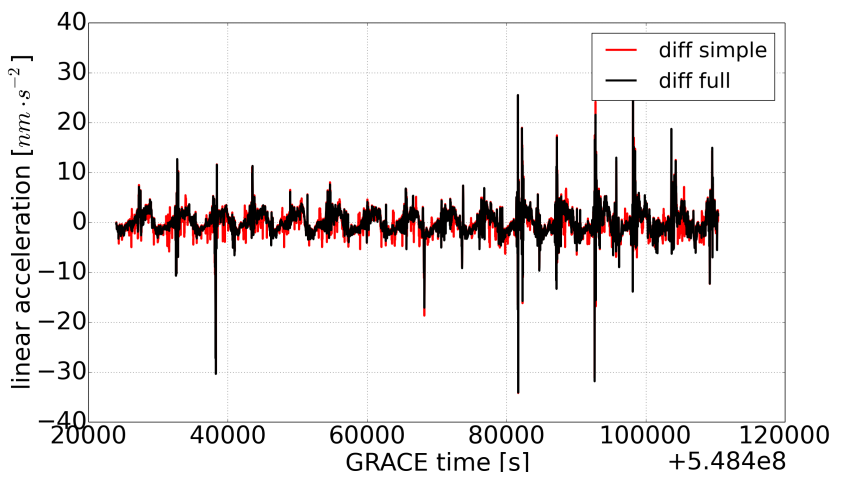

(a) along-track

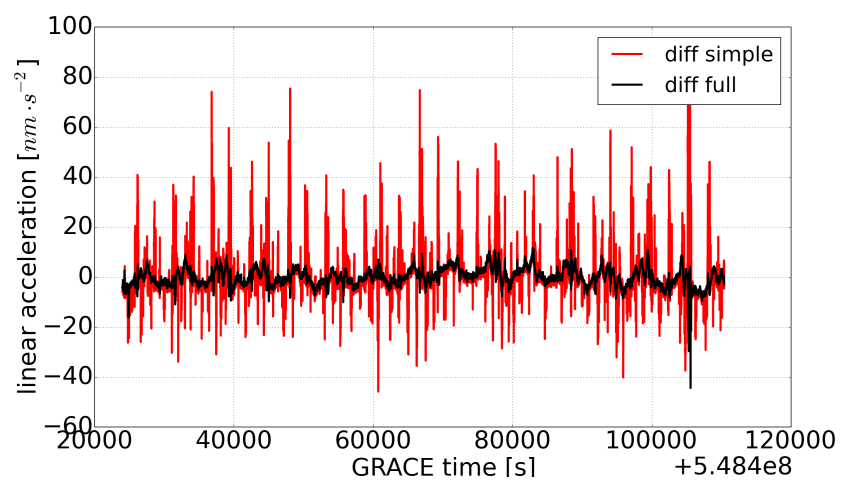

(c) cross-track

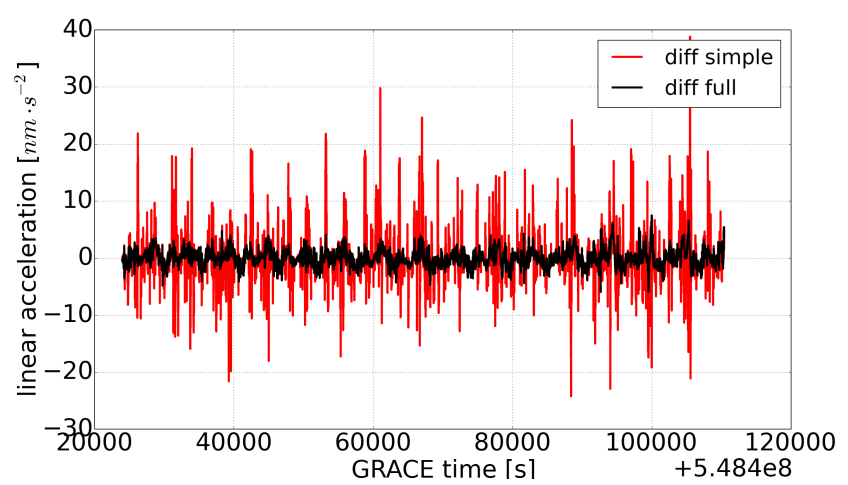

(e) radial

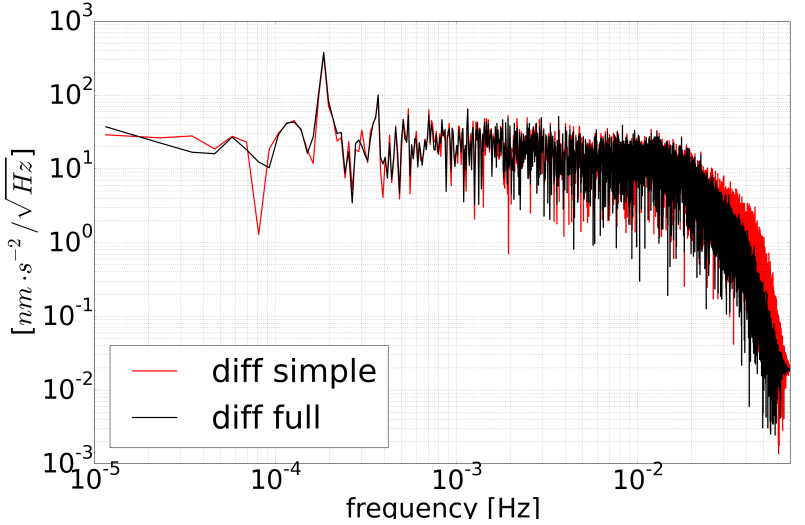

(b) along-track

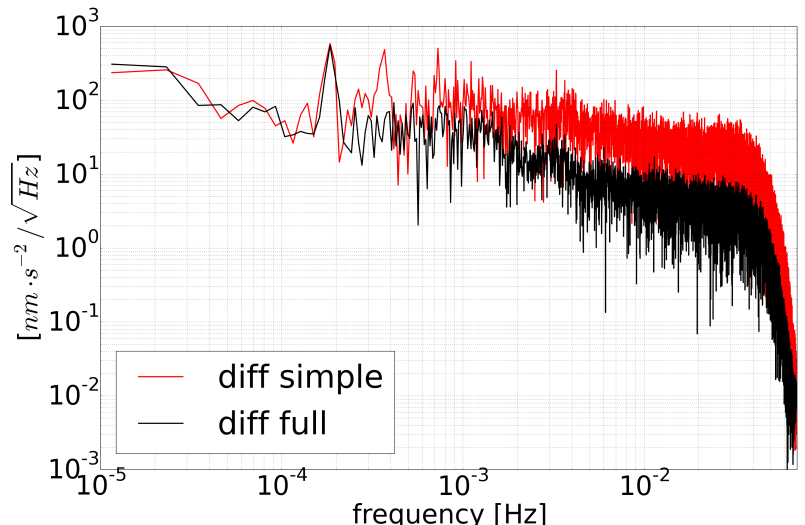

(d) cross-track

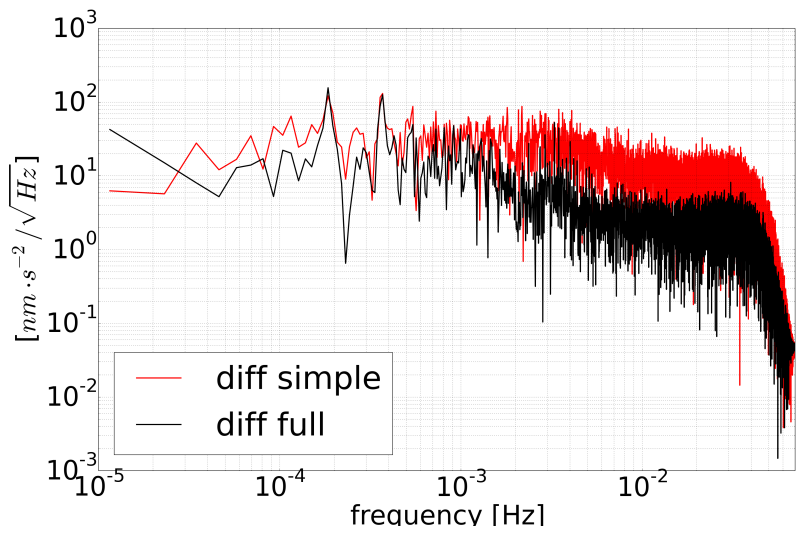

(f) radial

Figure 24: Comparison of differences of the transplanted linear acceleration and the original Level-1B data as measured by GRACE-B accelerometer on 2017-05-19 in both time domain (left column) and frequency domain (right column). The data obtained by "full" ACC data transplant (black curve) are characterized by significantly reduced high frequency noise compared to the data obtained by "simple" ACC transplant. This is a result of the applied thruster spike correction 


\subsection{Gravity field models}

To see the effect on the gravity field, we compared monthly gravity field solutions based on the three data sets: "simple" and "full" ACC data transplant and the original GRACE-B measurement. Figure 25 shows the degree variances and Figure 26 shows the global maps of geoid heights for the three solutions. The gravity field models were processed according to JPL RL05 standards (Watkins and Yuan, 2014). The geoid heights are shown relative to the nominal background field (GIF48), the solution was smoothed by a Gaussian filter with $400 \mathrm{~km}$ radius.

The "full" ACC data transplant results in significantly reduced noise for degrees beyond the first orbital resonance, when compared to the solution based on "simple" ACC data transplant. RMS difference of the solution based on transplanted ACC data relative to the solution based on original GRACE-A and GRACE-B ACC measurement are:

"Simple" transplant: $4.94 \mathrm{~cm}$ of EWH

"Full" transplant: $\quad 3.92 \mathrm{~cm}$ of EWH

which means the "full" ACC data transplant improves the gravity field error by approx. $1 \mathrm{~cm}$ of EWH (after smoothing to $400 \mathrm{~km}$ ) compared to the "simple" ACC data transplant.

The comparison of both the ACC timeseries and the gravity field solutions clearly proves that the "full" ACC data transplant provides significantly improved solution compared to the "simple" ACC data transplant, which is currently used as part of official processing algorithms for RL05. The "full" ACC data transplant, however, cannot fully substitute the actual ACC measurement itself. For the precise gravity field determination we still need working accelerometers onboard each spacecraft.

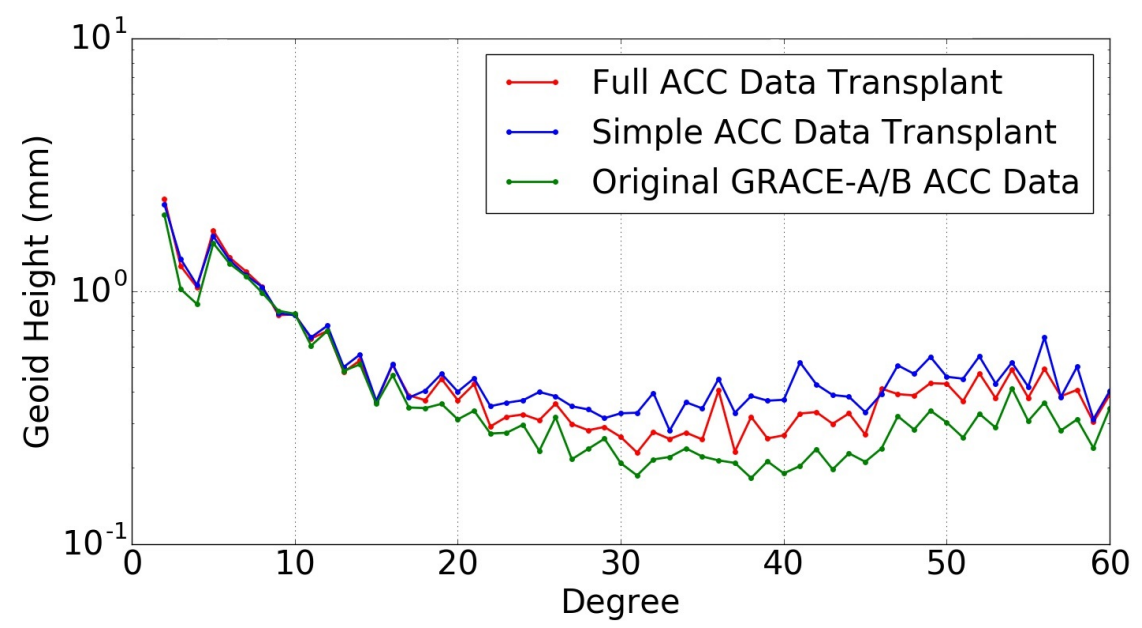

Figure 25: Comparison of the gravity field solutions based on the three ACC data sets in terms of degree variances for May 2017. No smoothing applied 


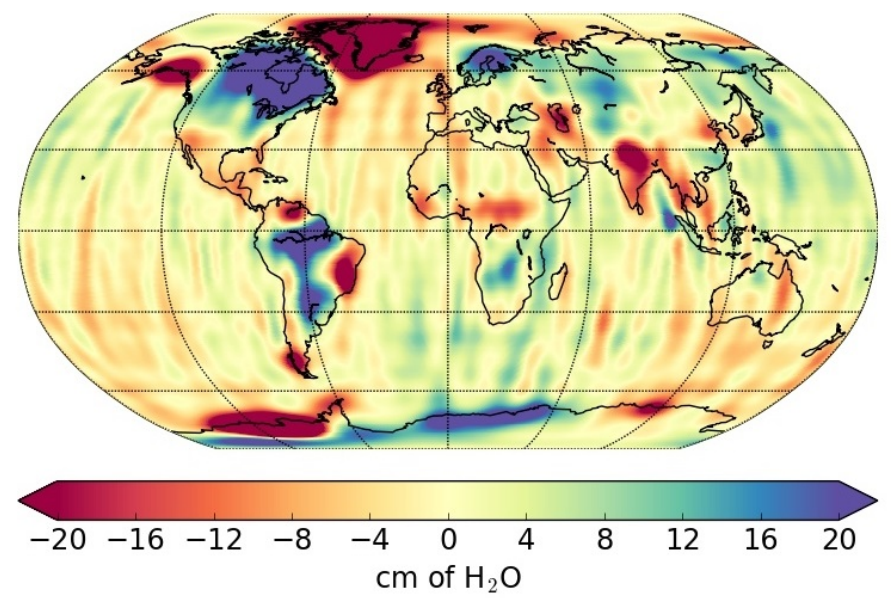

(a) original GRACE-B data (2 ACC solution)

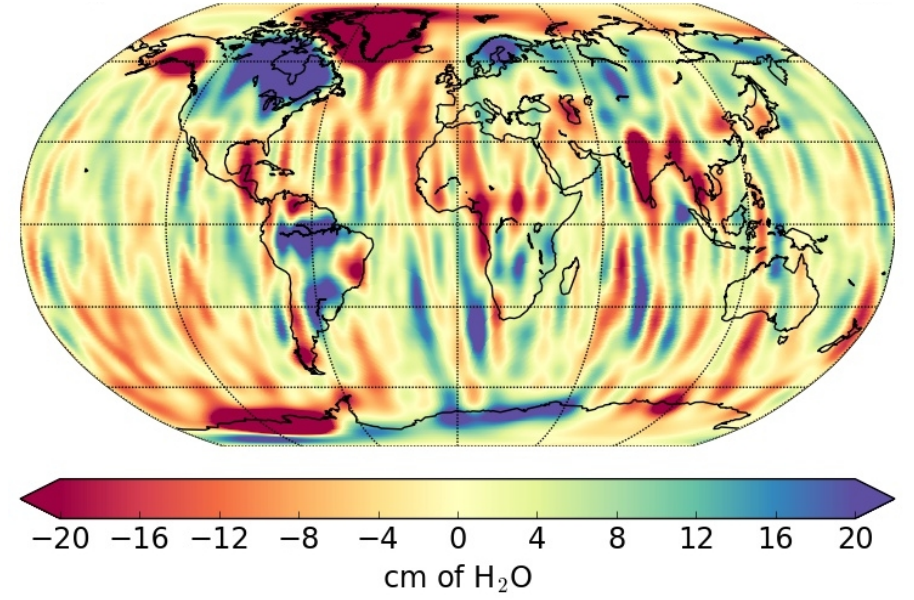

(b) "simple" ACC data transplant

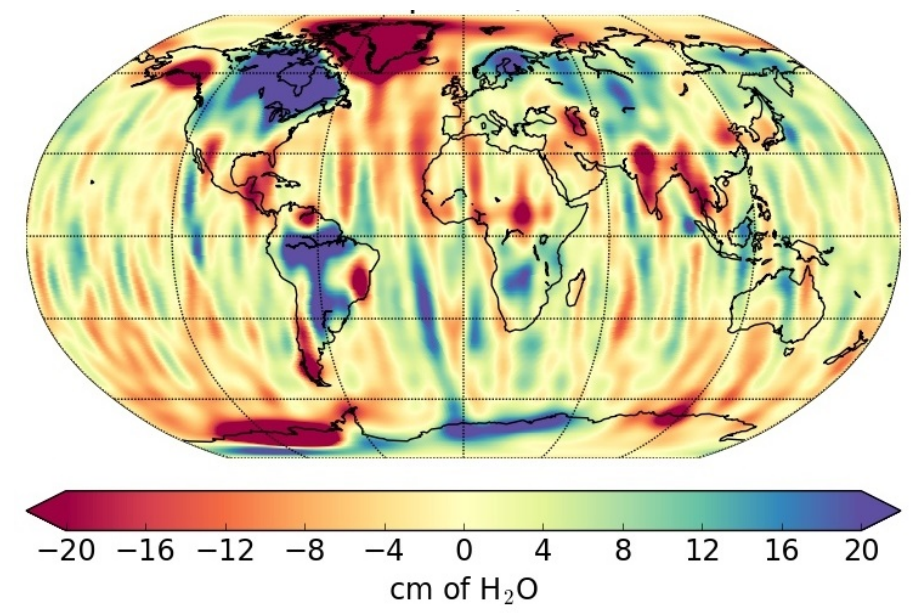

(c) "full" ACC data transplant

Figure 26: Monthly gravity fields (May 2017) based on the three ACC data sets in terms of geoid height relative to the nominal background field (GIF48), smoothed by a Gaussian filter with $400 \mathrm{~km}$ radius

\section{Gravity field solution for the months with missing GRACE-B ACC data}

The ultimate goal of the ACC data transplant is to generate the GRACE-B ACC timeseries for the months when the accelerometer was turned off in order to obtain the gravity field solution for these months. In the following, we present the comparison of the monthly gravity field models based on the ACC data obtained by "simple" and "full" ACC data transplant. The exact days for the monthly solutions are:

2016-11: Nov $14--$ Dec 10

2016-12: Dec 11 - Jan 6

2017-01: Jan 7 - Feb 3

2017-03: Mar 17 - Apr 14

2017-04: Apr 10 - May 8

2017-06: May 23 - Jun 29

Several days are excluded from the data analysis because of the ongoing satellite swap maneuver and battery issues, during which no K-band ranging data are available: Sep 4 - Nov 13, 2016 and Feb 4 - Mar 16, 2017.

Gravity field models were computed based on JPL RL05 standards. Figure27 shows the comparison of degree variances (with respect to the nominal background field GIF48) of both solutions for the respective months. As expected, the overall performance of the "full" ACC data transplant is significantly better compared to the "simple" transplant. The "full" ACC data transplant results in significantly decreased noise in degrees beyond the first orbital resonance. 
The improvement for June 2017 seems to be smaller than for the other months. This might be caused by the fact that there were many days with long thruster firings occurring simultaneously. The thruster model for these periods is not accurate. In addition, the pitch offset of GRACE-B significantly changed for 5 days (cf. Figure 13), which, for the sake of consistency, was not taken into account in the applied attitude correction.

Overall, we show that the gravity field solution based on the "full" ACC data transplant is characterized by significantly lower noise compared to the solution based on "simple" ACC transplant. It will be implemented as part of the official processing for the upcoming JPL RL06. Also, the new ACC data will be released as L1B product for November 2016 to June 2017.

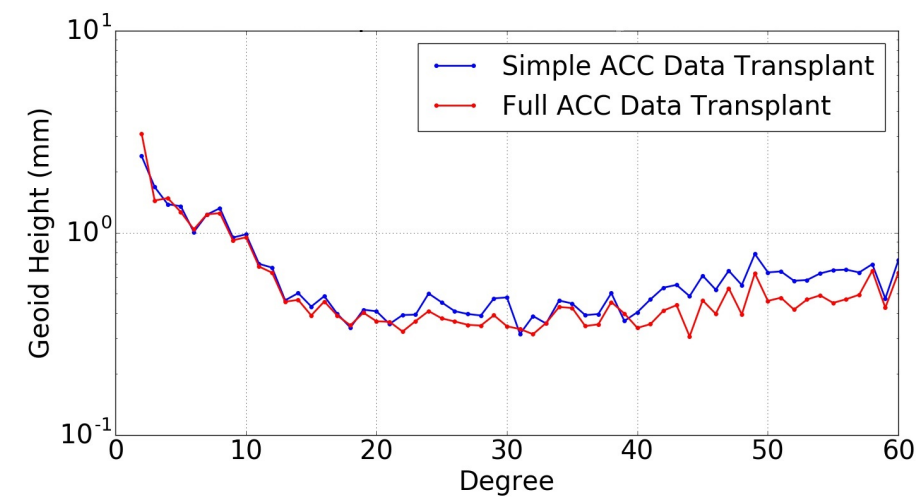

(a) November 2016

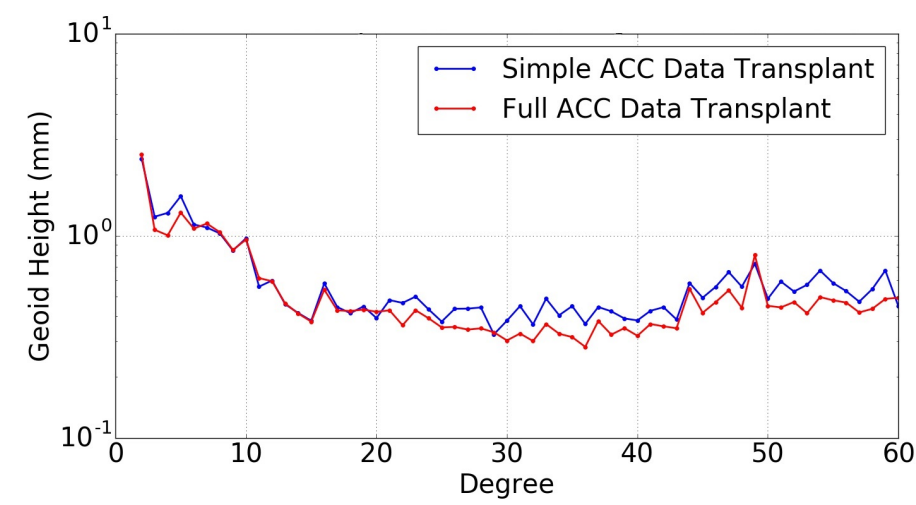

(c) January 2017

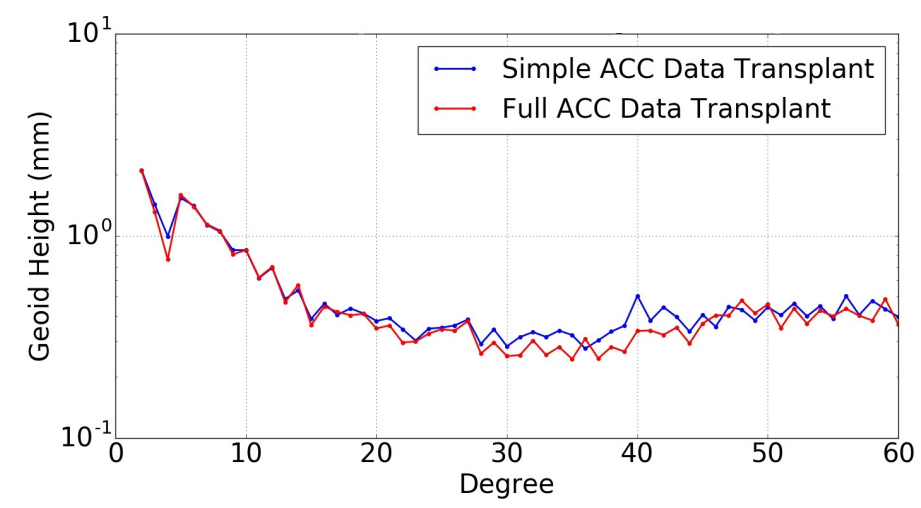

(e) April 2017

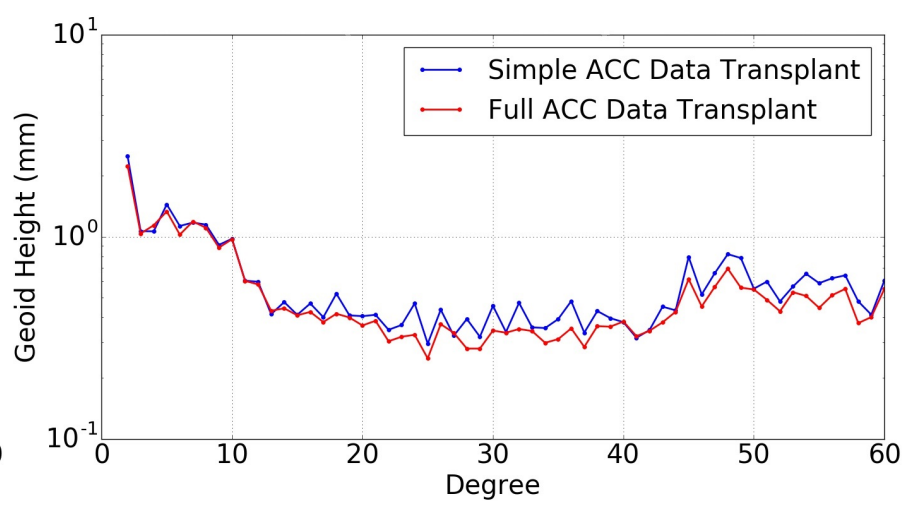

(b) December 2016

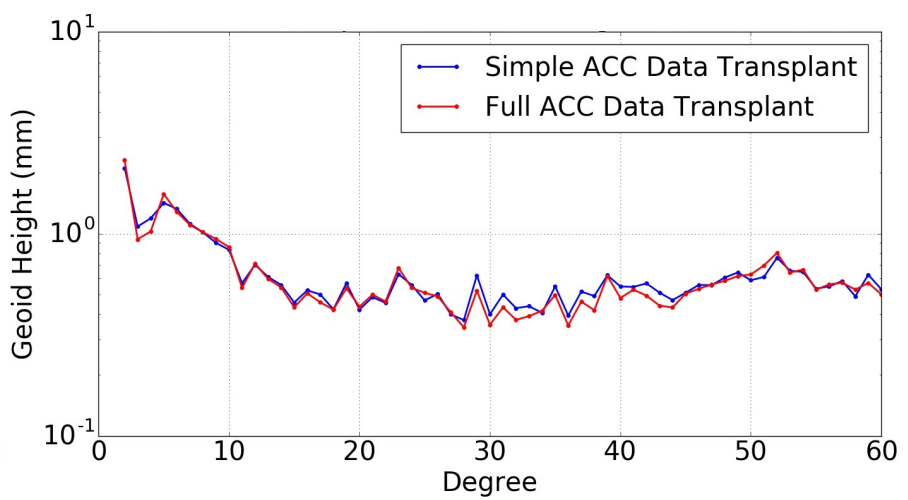

(d) March 2017

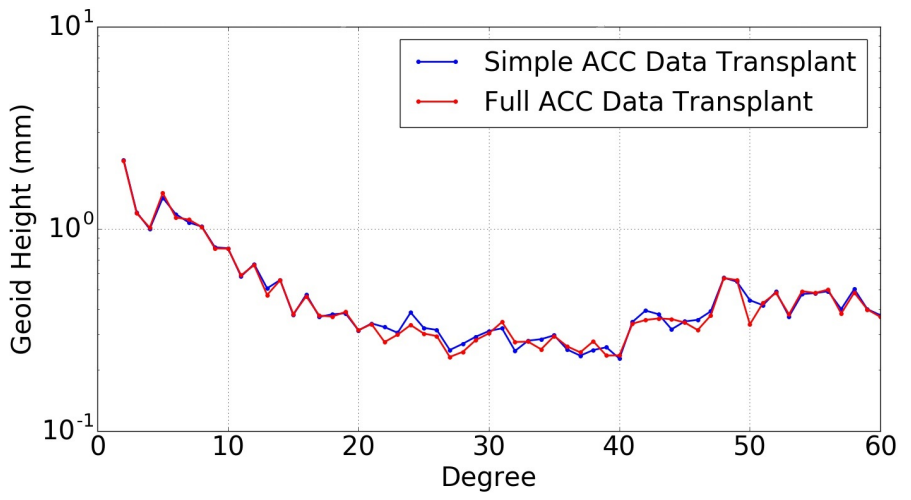

(f) June 2017

Figure 27: Comparison of the gravity field solutions based on the "simple" and "full" ACC data transplant in terms of degree variances (with respect to GIF48) for the months with missing GRACE-B ACC measurement. No smoothing applied 


\section{Conclusions}

The accelerometer data transplant allows to exploit the measurements of the GRACE mission in its final phase, despite the limitations caused by aging of the spacecraft. The fundamental idea of the ACC data transplant is to use GRACE-A ACC measurement to generate the missing GRACE-B ACC data. Using the ACC data transplant we can successfully recover the gravity field models for the months with single ACC operation (November 2016 June 2017).

A simple method for ACC data transplant method was already introduced more then 10 years ago, which was based only on attitude and time correction. In this paper, we presented an improved method for GRACE accelerometer data transplant based on modeling of the residual linear accelerations due to thruster firings. Our model is based on the actual characteristics of the ACC sensor as it is analytically derived from the ACC transfer function. By using the high rate $10 \mathrm{~Hz} \mathrm{ACC}$ data we ensure precise modeling of the thruster spikes.

Our ACC data transplant method results in significantly improved gravity field models characterized by lower noise for degrees beyond the first orbital resonance, compared to the simple method, which does not include the thruster spike correction. We demonstrated that the improvement of the gravity field solution was achieved for all months with missing GRACE-B measurement. For this reason, the improved ACC data transplant is now part of the processing standards for the upcoming JPL RL06 solution.

Despite the fact that ACC data transplant provides very good results for the months with missing ACC measurement, it cannot fully substitute the actual measurement itself. For precise gravity field modeling, data from both accelerometers are necessary.

\section{Acknowledgements}

The research was carried out at the Jet Propulsion Laboratory, California Institute of Technology, under a contract with the National Aeronautics and Space Administration. We would like to thank all reviewers for their valuable insight and for helping us to improve the quality of this publication.

\section{References}

\section{References}

Bettadpur, S. (2012). Gravity Recovery and Climate Experiment: Product specification document. Technical Report GRACE 327-720, Center for Space Research, The University of Texas at Austin.

Flury, J., Bettadpur, S., and Tapley, B. (2008). Precise accelerometry onboard the GRACE gravity field satellite mission. Advances in Space Research, 42, Pages 1414-1423, doi: $10.1016 /$ j.asr.2008.05.004

Frommknecht, B. (2008). Integrated Sensor Analysis of the GRACE Mission. PhD thesis, Technische Universität München, https://mediatum.ub.tum.de/doc/630607/454947.pdf

Herman, J., Presti, D., Codazzi, A., and Belle, C. (2004). Attitude control for GRACE: the first low-flying satellite formation. In ESA SP-548: 18th International Symposium on Space Flight Dynamics.

Herman, J., Davis, A., Chin, K., Kinzler, M., Scholz, S., and Steinhoff, M. (2012). Life with a weak heart - prolonging the GRACE mission despite degraded batteries. In SpaceOps 2012, Stockholm, Sweden, June 11-15.

Kang, Z., Tapley, B., Bettadpur, S., Ries, J., and Nagel, P. (2006). Precise orbit determination for GRACE using accelerometer data. Advances in Space Research, 38, Pages 2131-2136, doi: 10.1016/j.asr.2006.02.021

Klinger, B. and Mayer-Gürr, T. (2014). Combination of GRACE star camera and angular acceleration data. Geophysical Research Abstracts, 16, Pages EGU2014-5340.

Klinger, B. and Mayer-Gürr, T. (2016). The role of accelerometer data calibration within GRACE gravity field recovery: Results from ITSG-Grace2016. Adv Space Res, 58, Pages 1597-1609, doi: 10.1016/j.asr.2016.08.007

McCullough, C., Bettadpur, S., and McDonald, K. (2015). Accuracy of Numerical Algorithms for Satellite Orbit Propagation and Gravity Field Determination. Journal of spacecraft and rockets, 52/3, Pages 766-775, doi: 10.2514/1.A33008

Meyer, U., Jäggi, A., and Beutler, G. (2012). The Impact of Attitude Control on GRACE Accelerometry and Orbits. In K. et al. (Ed.), Geodesy for Planet Earth, IAG Symp., 136, Pages 139-146. Springer, doi: 10.1007/978-3-642-20338-1_17. 
Peterseim, N. (2014). Twangs - high-frequency disturbing signals in $10 \mathrm{~Hz}$ accelerometer data of the GRACE satellites. PhD thesis, Technische Universität München, https://mediatum.ub.tum.de/doc/1197563/1197563.pdf

Peterseim, N., Flury, J., and Schlicht, A. (2012). Magnetic torquer induced disturbing signals within GRACE accelerometer data. Advances in Space Research, 49/9, Pages 1388-1394, doi: 10.1016/j.asr.2012.02.013

Sakumura, C., Harvey, N., and Bandikova, T. (2017). The V03 Level-1 release. GRACE Science Team Meeting, Austin, TX, Oct 10-12, $201 \%$.

Save, H. (2016). GRACE battery status and science impact. GRACE Science Team Meeting, Potsdam, Germany Oct 5-7, 2016.

Save, H., Bettadpur, S., and Tapley, B. (2006). Single Accelerometer Gravity Solutions for GRACE. AGU Fall Meeting 2006, abstract G13A-0026.

Schelkle, M. (2000). The GRACE cold gas attitude and orbit control system. In 3rd International conference on spacecraft propulsion, ESA SP-465.

Tapley, B., Bettadpur, S., Watkins, M., and Reigber, C. (2004). The Gravity Recovery and Climate Experiment: Mission overview and early results. Geophysical Research Letters, 31, doi: 10.1029/2004GL019920

Touboul, P., Foulon, B., Willemenot E. (1999a). Electrostatic space accelerometers for present and future missions Acta Astronautica, 45/10, Pages 605-617, doi: 10.1016/S0094-5765(99)00132-0

Touboul, P., Willemenot E., Foulon, B., Josselin V. (1999b). Accelerometers for CHAMP, GRACE and GOCE space missions: synergy and evolution Bollettino di Geofisica Teorica ed Applicata, 40/3-4, Pages 321-327.

Touboul, P., Foulon, B., Rodrigues, M., and Marque, J. (2004). In orbit nano-g measurements, lessons for future space missions. Aerospace Science and Technology, 8/5, Pages 431-441, doi: 10.1016/j.ast.2004.01.006.

Tregoning, P., McClusky, S., Purcell, A., and Lescarmontier, L. (2013). Innovative analysis constraints in the ANU GRACE mascon solutions. AGU Fall Meeting 2013, abstract G32A-05.

Wang, F., Bettadpur, S., Save, H., and Kruizinga, G. (2010). Determination of Center-of-Mass of Gravity Recovery and Climate Experiment satellites. Journal of Spacecraft and Rockets, 47/2, Pages 371-379, doi $10.2514 / 1.46086$.

Watkins, M. and Yuan, D.-N. (2014). GRACE JPL Level-2 Processing Standards Document for Level-2 Product Release 05.1. Technical Report GRACE 327-744 (v 5.1), Jet Propulsion Laboratory.

Wiese, D., McCullough, C., Yuan, D.-N., Kuang, D., Boening, C., and Landerer, F. (2017). GRACE JPL Level 2 processing status. GRACE Science Team Meeting, Austin, TX, Oct 10-12, $201 \%$.

Witkowski, M. and Massmann, F.-H. (2016). Status GRACE mission operations. GRACE Science Team Meeting, Potsdam, Germany, Oct 5-7, 2016.

Witkowski, M. and Massmann, F.-H. (2017). Status GRACE mission operations. GRACE Science Team Meeting, Austin, TX, Oct 10-12, 2017.

Wolfram Research, Inc. (2016). Mathematica, version 11.0.1 (student edition). Champaign, IL, 2016.

Wu, S.-C., Kruizinga, G., and Bertiger, W. (2006). Algorithm theoretical basis document for GRACE Level-1B data processing v1.2. Technical Report GRACE 327-741, Jet Propulsion Laboratory. 